\title{
An Effective Sample Size for Predicting Plant Disease Incidence in a Spatial Hierarchy
}

\author{
L. V. Madden and G. Hughes
}

First author: Department of Plant Pathology, Ohio State University, Wooster 44691-4096; and second author: Institute of Ecology and Resource Management, University of Edinburgh, West Mains Road, Edinburgh EH9 3JG, Scotland.

Accepted for publication 24 May 1999.

ABSTRACT

\begin{abstract}
Madden, L. V., and Hughes, G. 1999. An effective sample size for predicting plant disease incidence in a spatial hierarchy. Phytopathology 89 : 770-781.

For aggregated or heterogeneous disease incidence, one can predict the proportion of sampling units diseased at a higher scale (e.g., plants) based on the proportion of diseased individuals and heterogeneity of diseased individuals at a lower scale (e.g., leaves) using a function derived from the beta-binomial distribution. Here, a simple approximation for the betabinomial-based function is derived. This approximation has a functional form based on the binomial distribution, but with the number of individuals per sampling unit $(n)$ replaced by a parameter $(v)$ that has similar interpretation as, but is not the same as, the effective sample size $\left(n_{\text {deff }}\right)$ often used in survey sampling. The value of $v$ is inversely related to the
\end{abstract}

degree of heterogeneity of disease and generally is intermediate between $n_{\text {deff }}$ and $n$ in magnitude. The choice of $v$ was determined iteratively by finding a parameter value that allowed the zero term (probability that a sampling unit is disease free) of the binomial distribution to equal the zero term of the beta-binomial. The approximation function was successfully tested on observations of Eutypa dieback of grapes collected over several years and with simulated data. Unlike the beta-binomial-based function, the approximation can be rearranged to predict incidence at the lower scale from observed incidence data at the higher scale, making group sampling for heterogeneous data a more practical proposition.

Additional keywords: dispersion, models, quantitative epidemiology, spatial pattern.
There are many uses for information on the spatial heterogeneity of disease incidence in relation to sampling methodology $(14,20$, $29,35)$, comparison of treatments $(17,23,45)$, prediction of yield loss $(8,12,18,31)$, and assessment of spatiotemporal disease dynamics $(9,10,22,30,33)$. Hughes et al. (21) recently showed an additional use of spatial heterogeneity: once mean incidence is (precisely) determined at some lower level in a spatial hierarchy (e.g., leaf), prediction of incidence at a higher level (e.g., plant) is dependent on the degree of heterogeneity of incidence at the lower scale. In particular, as the degree of aggregation increases at the lower scale (at a given mean incidence), the incidence of disease at the higher scale decreases. This relationship between incidence at two levels can be exploited to improve sampling methods $(13,14)$ and to understand the temporal dynamics of disease at multiple hierarchical levels $(21,27,33,43)$.

Characterization of spatial heterogeneity depends on the methods used for collecting data on diseased and healthy individuals $(2,22)$. If cluster sampling is used-in which $N$ sampling units are obtained (randomly or with some other approach) and the disease status of every individual within the sampling units (groups) is determined (25) - then it is straightforward to represent the data using discrete statistical distributions for random or overdispersed data. One can quantify the degree of aggregation using parameters of these discrete distributions $(22,42)$. There is now ample evidence that the beta-binomial distribution (16) provides a very good description of populations of diseased individuals in many pathosystems $(16,19,26-30,37,40,41,44,45)$. A limiting case of the beta-binomial is the binomial distribution, which can be used to describe random patterns of incidence (27).

Corresponding author: L. V. Madden; E-mail address: MADDEN.1@osu.edu

Publication no. P-1999-0709-01R

(C) 1999 The American Phytopathological Society
Functional relationships between disease incidence at two levels in a spatial hierarchy can be derived based on the beta-binomial and binomial distributions (21). Using these functions, incidence of disease at the higher level can be predicted, without curve fitting, based solely on the incidence and degree of aggregation at the lower level. If incidence is binomially distributed (random), one can take the reciprocal approach and predict incidence at the lower level based on incidence at the higher level. This is particularly useful in the context of group testing and sampling $(7,39)$, in which the disease status of each group (e.g., sampling unit) is determined, possibly with a biochemical test of the pooled (grouped) individuals (13), and the results from the sampling units are used to calculate the proportion of diseased individuals at the lower level. This group testing approach is known to be very efficient for estimating incidence at the lower level under many circumstances $(11,39)$. For incidence data that have a beta-binomial distribution (overdispersed or aggregated data at some spatial scale), this approach is not practical, because the beta-binomial function generally cannot be rearranged to predict incidence at the lower level based on the higher-level incidence. Some empirical methods, based on curve fitting, have been found to be useful for specific pathogenhost systems when the data were overdispersed (14). Because it is more likely that disease incidence data will be described by the beta-binomial than by the binomial distribution $(16,19,27,30,41)$, research was conducted to determine a function that is easier to use than the beta-binomial-based one for relating incidence at the two levels based on the known or assumed spatial heterogeneity.

The purpose of this study was to further explore the relationship between disease incidences at two levels in a spatial hierarchy, with the goal of finding a simple approximation to interrelate incidence at the two levels. In this context, we considered use of an effective sample size, as defined by Kish (25), or similar statistic. The effective sample size as defined by Kish (25) is an adjustment of the group size for overdispersed data that has long been used in survey sampling. Rao and Scott (36) have recently shown that use 
of an effective sample size allows one to transform overdispersed incidence data and then, for certain types of statistical tests, analyze the data as if they were binomially distributed. This avoids the complexities of maximum likelihood methods for beta-binomial distributions, which typically require specialized computer software. To develop the approximation shown in this paper, we considered ancillary ways of graphically and functionally relating incidence at two levels in order to determine a new effective sample size statistic. Although the motivation for this work was practical, it is shown how the research leads to an expanded understanding of the spatial-level problem. The work was based on the assumption that the beta-binomial is an accurate representation of actual disease incidence data, which we believe is reasonable $(22,27)$. Theoretical results are supported by an analysis of experimental data (34) and a Monte Carlo simulation.

\section{THEORY}

Spatial levels. We consider there to be $N$ sampling units (groups), $n$ individuals per sampling unit, and $X$ diseased individuals per sampling unit. For example, there are $X_{j}(j=1, \ldots, N)$ diseased individuals in the $j$ th sampling unit. Sampling units could be groups of plants (e.g., quadrats), plants, or plant units (e.g., branches); individuals could be plants (e.g., $n$ plants per sampling unit [quadrat]), leaves (e.g., $n$ leaves per sampling unit [plant]), or other plant units. For simplicity, we consider $n$ to be the same for all sampling units. Using the symbols in Hughes et al. (21), we define $p_{\text {Low }}$ as the expected probability that an individual at the lower level in a hierarchy is diseased; $p_{\text {Low }}$ can be estimated directly from information on diseased individuals. In particular, the moment estimate of $p_{\text {Low }}$ ( $\hat{p}_{\text {Low }}$ ) is the proportion of the $n \cdot N$ individuals that are diseased. We then define $p_{H i g h}$ as the probability that a sampling unit has one or more diseased individuals; $p_{\text {High }}$ could be directly estimated as the proportion of sampling units with one or more diseased individuals $\left(\hat{p}_{\text {High }}\right)$. The zero term of the statistical distribution of $X$ is $\operatorname{Pr}(X=0)=1-p_{\text {High }}$; this represents the probability that a sampling unit has no diseased individuals.

If the binomial distribution is appropriate, the relationship between disease incidence at the two levels can be written as

$$
p_{\text {High }}=1-\left(1-p_{\text {Low }}\right)^{n}
$$

If $X$ has a beta-binomial distribution, the relationship can be written as

$$
p_{\text {High }}=1-\prod_{i=0}^{n-1} \frac{1-p_{\text {Low }}+i \theta}{1+i \theta}
$$

in which $\Pi$ is the product function, $i$ is an index of integers from 0 to $n-1$, and $\theta$ is the aggregation parameter of the distribution. The moment estimate of $\theta$ is given by (16)

$$
\hat{\theta}=\frac{S^{2}-n \hat{p}_{\text {Low }}\left(1-\hat{p}_{\text {Low }}\right)}{n^{2} \hat{p}_{\text {Low }}\left(1-\hat{p}_{\text {Low }}\right)-S^{2}}
$$

in which $S^{2}$ is the calculated variance of $X$ (in which each value is an integer in the range 0 to $n$ ), and $\hat{p}_{\text {Low }}$ is the estimated $p_{\text {Low }}$. Maximum likelihood estimation, which is an iterative procedure, also can be done with specialized software (26) or with some commercial products (e.g., Egret; Cytel Software Corp., Cambridge, MA). When $\theta=0$, the beta-binomial distribution reduces to the binomial, and equation 2 is then equivalent to equation $1 \mathrm{a}$.

A note on symbols for parameter estimates is needed here. Following Hughes et al. (21), we use a 'hat' to indicate an estimate made with data at the same spatial level (e.g., $\hat{p}_{H i g h}$ for the proportion of sampling units with one or more diseased individuals). We use a 'tilde' to indicate an estimate of a parameter at one level based on data at another spatial level (e.g., $\widetilde{p}_{\text {High }}$ for estimated $p_{\text {High }}$ based on a function of the proportion of diseased individuals $\left[\hat{p}_{\text {Low }}\right]$ ). When referring to terms conceptually or theoretically, we use symbols without hats or tildes.
Equations 1a and 2 both represent saturation-type curves, in which $p_{\text {High }}$ rises monotonically to 1 as $p_{\text {Low }}$ increases (Fig. 1A). The steepest curve (at low $p_{\text {Low }}$ ) is for the binomial case (eq. 1a), and increasing aggregation leads to less steep curves (especially at small $p_{\text {Low }}$ ). As pointed out in Hughes et al. (21), for any given value of $p_{\text {Low }}$, the value of $p_{\text {High }}$ decreases as $\theta$ increases (Fig. 1A). If aggregation of disease incidence is a function of $p_{\text {Low }}$, such that the binary form of the power law describes the results (15), then one can expand equation 2 by making $\theta$ a function of $p_{\text {Low }}(27,33)$ (discussed below). Equation 2 was found to describe several data sets without any formal curve fitting, once information on $p_{\text {Low }}$ and $\theta$ was available (21).
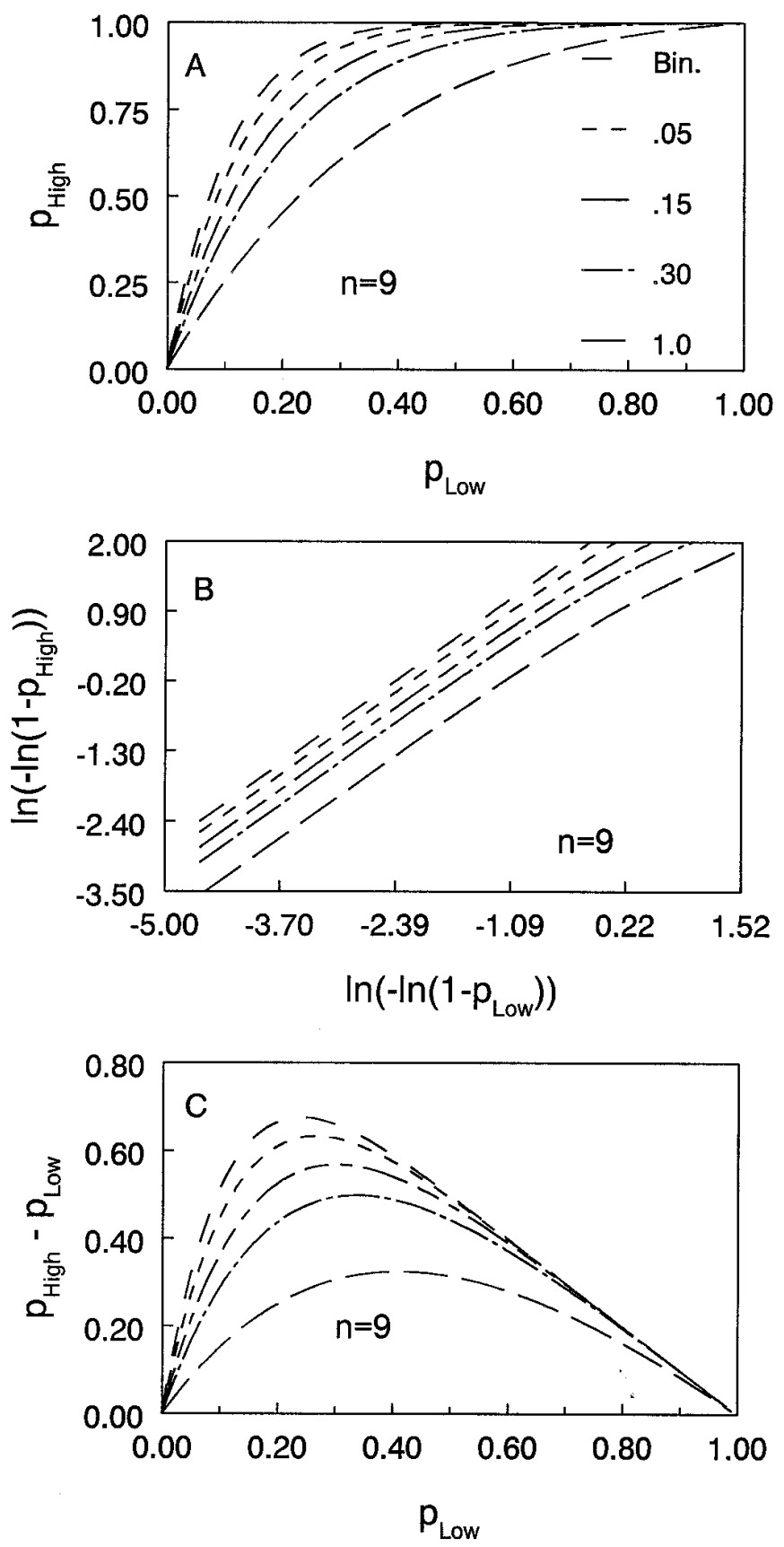

Fig. 1. Relationship between incidence of disease at two levels in a spatial hierarchy. A, Expected probability of individuals (e.g., leaves) being diseased $\left(p_{\text {Low }}\right)$ in relation to the probability of a sampling unit (e.g., plant) having one or more diseased individuals $\left(p_{\text {High }}\right)$ at selected values of the aggregation parameter $(\theta)$ of the beta-binomial distribution (eq. 2). 'Bin.' is for the binomial distribution $(\theta=0$ [eq. 1a]). All curves are for $n=9$; $\theta$ increases as one moves from the top to the bottom curves. B, Complementary log-log transformations of $p_{\text {Low }}$ and $p_{\text {High }}$ in $\mathbf{A}$ (eq. 5a). C, Relation between $p_{\text {Low }}$ and the difference between $p_{H i g h}$ and $p_{\text {Low }}(\Delta p)$ in $\mathbf{A}$ (eqs. 6a and 7). 
If one wished to predict $p_{\text {Low }}$ based on $p_{\text {High }}$ for the binomial distribution, this would be accomplished by rearranging equation 1a to

$$
p_{\text {Low }}=1-\left(1-p_{\text {High }}\right)^{1 / n}
$$

which is the basis for group testing in many disciplines $(11,39)$. If one estimated $p_{\text {High }}$ directly with data $\left(\hat{p}_{\text {High }}\right)$, substitution of this estimate in equation 4 will produce $\widetilde{p}_{\text {Low }}$, an estimate of $p_{\text {Low }}$ based on disease incidence data at the higher scale. However, for data de-
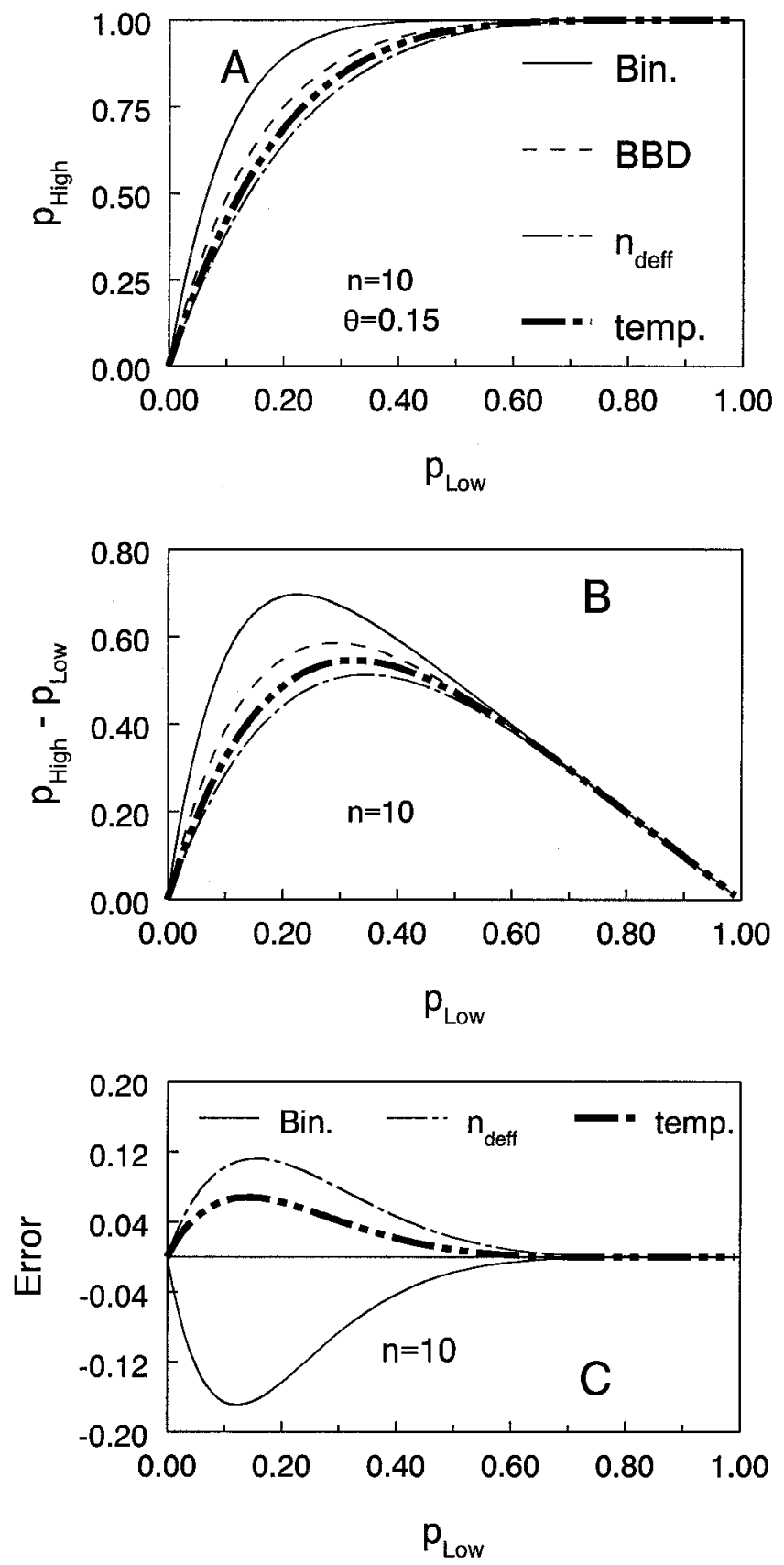

Fig. 2. Relationship between incidence of disease at two levels in a spatial hierarchy for a single value of the index of aggregation of the beta-binomial distribution $(\theta=0.15)$ and of the number of individuals per sampling unit $(n=$ $10)$ and various approximations for the relationship. 'BBD' is for the expected probability of individuals being diseased $\left(p_{\text {Low }}\right)$ in relation to the probability of a sampling unit having one or more diseased individuals ( $p_{\text {High }}$ ) (eq. 2 ), considered the true result here. 'Bin.' is for the binomial-based predictions (eq. 1a); ' $n_{\text {deff }}$ ' is for the predictions based on equation $1 \mathrm{~b}$ with $\varepsilon=\mathrm{n}_{\text {deff; }}$ ' 'temp.' is for the predictions based on equation $1 \mathrm{~b}$ with $\varepsilon$ being assigned a temporary (arbitrary) value of 5.2. A, $p_{\text {High }}$ versus $p_{\text {Low }}$. B, Relationship between $p_{\text {Low }}$ and the difference between $p_{\text {High }}$ and $p_{\text {Low }}(\Delta p)$ (eqs. 6a, 7, and 6b). C, Error in predicting $p_{\text {High }}$ of the BBD curve with $n_{\text {deff }}$, Bin.-, or temp.-based curves. scribed by equation 2 , it is not generally possible to find a rearranged equation giving $p_{\text {Low }}$ as a function of $p_{\text {High }}$. The only exceptions are for very small and even $n$ (e.g., $n=2$ and $n=4$ [eq. 5 in literature citation 21]). Nevertheless, visual inspection of Figure 1A shows a similarity in shape of the curves for different nonzero $\theta$ values, implying that approximations for these curves might be possible without the need of the complicated equation 2 . This can be further seen from the transformation shown in the next section.

Complementary log-log (CLL) function. As shown by Farrington (7), one can transform equation 1a to

$$
\ln \left[-\ln \left(1-p_{\text {High }}\right)\right]=\ln (n)+\ln \left[-\ln \left(1-p_{\text {Low }}\right)\right]
$$

in which $\ln [-\ln (1-\bullet)]$ is known as the CLL transformation function. A plot of $\ln \left[-\ln \left(1-p_{\text {High }}\right)\right]$ versus $\ln \left[-\ln \left(1-p_{\text {Low }}\right)\right]$ is a straight line with slope of 1 and an intercept of $\ln (n)$ (Fig. 1B). The $\ln (n)$ term is a measure of the height of the line. Rearrangement of equation 2 for the beta-binomial distribution in the form of the CLL equation produces

$$
\ln \left[-\ln \left(1-p_{\text {High }}\right)\right]=\ln \left(-\sum_{i=0}^{n-1} \ln \frac{1-p_{\text {Low }}+i \theta}{1+i \theta}\right)
$$

which does little to clarify the situation, since the right-hand side of the equation is the function of a relatively complicated summation. However, if one plots $\ln \left[-\ln \left(1-p_{\text {High }}\right)\right]$ versus $\ln [-\ln (1-$ $\left.\left.p_{\text {Low }}\right)\right]$ for the generated beta-binomial curves in Figure 1A, one can see that nearly straight lines result over most of the range of $p_{\text {Low }}$, almost parallel to the binomial line of equation 5a (Fig. 1B). These lines would correspond, to a reasonable approximation, to equation 5 a with $\ln (n)$ replaced by a smaller constant. Only at large $p_{\text {High }}$ (e.g., $p_{\text {High }} \geq 0.8$ ), corresponding to $\operatorname{CLL}\left(p_{\text {High }}\right) \geq 0.5$, do the beta-binomial-based lines appreciably curve downward relative to the binomial-based lines. This curvature is most evident only for very large $\theta$. Thus, for relating disease incidences at two spatial levels, there is evidence that one could approximately duplicate the beta-binomial function (right-hand side of eq. 2) with an equation using the form of the binomial function (right-hand side of eq. 1a), with $n$ replaced by a smaller constant. The work of Rao and Scott (36) on effective sample size is in the spirit of this approach.

A difference function. A problem in working with saturationtype responses such as equations $1 \mathrm{a}$ and 2 is that differences in the shapes of the curves tend to be rather subtle, and visual comparisons of curves are, therefore, difficult to make. The CLL function (eqs. 5a and 5b) allows one to more clearly see the differences in $p_{\text {High }}$ over the range of $p_{\text {Low }}$, although there is the conceptual difficulty of plotting $\operatorname{CLL}\left(p_{\text {High }}\right)$ versus $\operatorname{CLL}\left(p_{\text {Low }}\right)$ (Fig. 1B) when the 'predictor' or explanatory variable in equation $5 \mathrm{~b}$ is actually a more complicated function of $p_{\text {Low }}$. A further difficulty that might arise when using the CLL function is that CLL(1) is undefined, so observed data for which all the sampling units have at least one diseased individual $\left(p_{\text {High }}=1\right)$ cannot easily be dealt with using this approach.

An alternate way of considering the spatial hierarchy curves of Figure $1 \mathrm{~A}$ is to subtract $p_{\text {Low }}$ from each side of equations $1 \mathrm{a}$ and 2. The new variable, which represents how much larger $p_{\text {High }}$ is compared with $p_{\text {Low }}$, is written as $\Delta p=p_{\text {High }}-p_{\text {Low }}$. One can write the equations for the binomial and beta-binomial functions (eqs. 1a and 2 , respectively) as

$$
\Delta p=1-\left(1-p_{\text {Low }}\right)^{n}-p_{\text {Low }}
$$

and

$$
\Delta p=1-\prod_{i=0}^{n-1} \frac{1-p_{\text {Low }}+i \theta}{1+i \theta}-p_{\text {Low }}
$$

These equations represent curves that originate at 0 for $p_{\text {Low }}=0$, rise to a maximum, and then decline to 0 at $p_{\text {Low }}=1$ (Fig. 1C). The value of $n$ influences the maximum $\Delta p$ and the value of $p_{\text {Low }}$ in 
which the maximum occurs. At relatively large $p_{\text {Low }}$ values (e.g., $\left.p_{\text {Low }} \geq 0.6\right), \Delta p$ decreases with $p_{\text {Low }}$ in a straight-line fashion, with a slope of -1 , because $p_{\text {High }} \approx 1$ at these large values of $p_{\text {Low }}$.

For the beta-binomial-based equation 7 , the value of $\theta$ influences the magnitude of $\Delta p$ and the value of incidence at the lower scale in which the maximum occurs. For all values of $\theta$ greater than 0 , $\Delta p$ of equation 6a (binomial) is greater than or equal to $\Delta p$ of equation 7 (beta-binomial). For instance, with $n=9$ and $\theta=0.30$, the maximum $\Delta p$ from equation 7 is 0.50 , which occurs at $p_{\text {Low }}=0.34$; at this $p_{\text {Low }}, \Delta p$ from equation $6 \mathrm{a}$ is 0.64 . The $\Delta p$ function facilitates comparison of binomial- and beta-binomial-based relationships between disease incidences at two levels in a spatial hierarchy. Later, use of $\Delta p$ in approximating a beta-binomial curve (eq. 2) with a curve in the form of the binomial (eq. 1a) is discussed.

Effective sample size. For over 3 decades, the concept of a design effect, deff, has been used with survey sampling of overdispersed binary data such as disease incidence (25). The deff is defined as the ratio of the observed variance of the proportions to the theoretical variance for a binomial distribution $\left(=p_{\text {Low }} \cdot\left(1-p_{\text {Low }}\right) / n\right)$. This is the same as the index of dispersion $(D)$ in the statistical ecology area (27). If the data are beta-binomially distributed, deff can be written as

$$
\text { deff }=\frac{1+n \theta}{1+\theta}
$$

Effective sample size is then given by

$$
n_{\text {deff }}=\frac{n}{d e f f}
$$

For overdispersed data, deff $>1$ and $n_{\text {deff }}<n$. The term 'effective sample size' is used to indicate that overdispersion-in the current context, the tendency for individuals in the same sampling unit to have the same disease status-results in less information being obtained than if the disease status of individuals in a sampling unit were independent of the disease status of other individuals in the same sampling unit (5) (discussed below). Kish (25) adopted $n_{\text {deff }}$ (in place of $n$ ) in order to be able to use formulae appropriate for simple random sampling when, in fact, some other form of sampling design had been used. More recently, Rao and Scott (36) used $n_{\text {deff }}$ (in place of $n$ ) in developing a simple method for the analysis of overdispersed binary data. For grouped binary data, use of the effective sample size concept is founded on the transformation of data so that the first and second moments (mean and variance) of the transformed data are of the binomial form (36).

With sample observations, one could obtain the moment estimate of $\theta$ based on the calculated deff, or one could estimate deff using an estimate of $\theta$. A deff of 1 is consistent with a binomial distribution, and increasing deff is a measure of increasing heterogeneity at a given level of $p_{\text {Low }}$.

The approximation problem. The successful use of $n_{\text {deff }}$ for various types of analyses suggests that $n_{\text {deff }}$, or perhaps another constant, could be used in a binomial-type function to interrelate $p_{\text {High }}$ and $p_{\text {Low }}$, avoiding the need for the rather complicated equation 2. Specifically, we attempted to approximate the beta-binomial-based equation 2 with an equation of the following form

$$
p_{\text {High }}=1-\left(1-p_{\text {Low }}\right)^{\varepsilon}
$$

in which $\varepsilon$ is a selected constant. Obviously, if $\varepsilon=n$, equation $1 \mathrm{~b}$ is identical to equation $1 \mathrm{a}$. If, in equation $2, \theta>0$, our stated objective requires a choice of $\varepsilon<n$. As indicated above, $n_{\text {deff }}$ fulfills this requirement. Use of $\varepsilon=n_{\text {deff }}$ in equation $1 \mathrm{~b}$ is, therefore, the starting point in investigating the extent to which a binomial-type function can mimic equation 2 .

Using Mathcad mathematics software (MathSoft, Inc., Cambridge, MA), the following terms were calculated for $0 \leq p_{\text {Low }} \leq 1$ : (i) $p_{\text {High }}$ based on equation 2 (beta-binomial) with any chosen values of $n$ and $\theta$; (ii) $p_{H i g h}$ based on equation $1 \mathrm{~b}$ with any chosen value of $\varepsilon$, although it is values $n_{\text {deff }} \leq \varepsilon \leq n$ (eq. 1a) that are of particular interest; (iii) $\Delta p$ from equation 7 (beta-binomial) and $\Delta p$ derived from equation $1 \mathrm{~b}$

$$
\Delta p=1-\left(1-p_{\text {Low }}\right)^{\varepsilon}-p_{\text {Low }}
$$

and (iv) the error involved in using equation $1 \mathrm{~b}$ to approximate $p_{\text {High }}$ values given by equation 2 for chosen values of $n$ and $\theta$. This
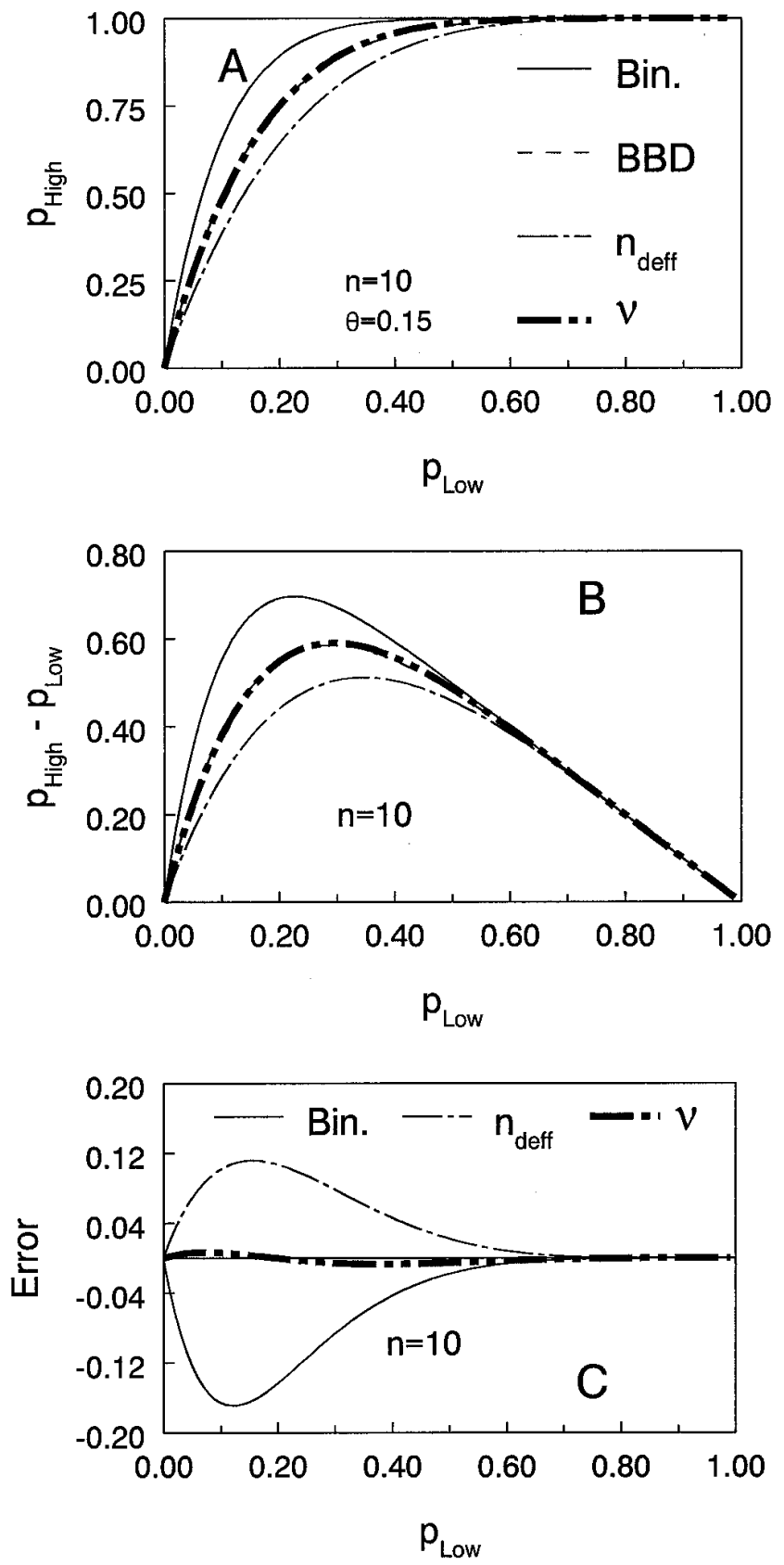

Fig. 3. Relationship between incidence of disease at two levels in a spatial hierarchy for a single value of the index of aggregation of the beta-binomial distribution $(\theta=0.15)$ and of the number of individuals per sampling unit $(n=$ $10)$ and various approximations for the relationship. 'BBD' is for the expected probability of individuals being diseased $\left(p_{\text {Low }}\right)$ in relation to the probability of a sampling unit having one or more diseased individuals $\left(p_{\text {High }}\right)$ (eq. 2 ), considered the true result here. 'Bin.' is for the binomial-based predictions (eq. 1a); ' $n_{\text {deff }}$ ' is for the predictions based on equation $1 \mathrm{~b}$ with $\varepsilon=n_{\text {deff }}$ ' ' $v$ ' is for the predictions based on equation 1c with $v=6.2$ (in which $v$ is the new or corrected effective sample size). This figure is identical to Figure 2, except for the $v$-based curves replacing the temp. curves. $\mathbf{A}, p_{H i g h}$ versus $p_{\text {Low }} . \mathbf{B}$, Relationship between $p_{\text {Low }}$ and the difference between $p_{\text {High }}$ and $p_{\text {Low }}(\Delta p)$ (eqs. 6a, 7, and 6b). C, Error in predicting $p_{H i g h}$ of the BBD curve with $n_{\text {deff- }}$, Bin.-, or $v$-based curves. 
error is calculated as the difference between $p_{\text {High }}$ based on equation 2 (beta-binomial) and $p_{H i g h}$ based on equation $1 \mathrm{~b}$ with any chosen value of $\varepsilon$ (although, again, it is values $n_{\text {deff }} \leq \varepsilon \leq n$ that are of particular interest). The Mathcad worksheet is available for free from the authors.

An example is shown in Figure 2A for $n=10$ and $\theta=0.15$ (deff $=$ 2.174 [eq. 8]). The values of $p_{\text {High }}$ for the beta-binomial (short dashes) are less than for the binomial (solid line, highest curve) at all values of $p_{\text {Low }}$. Use of $n_{\text {deff }}(10 / 2.174=4.6$ [eq. 9a]) in equation $1 \mathrm{~b}$ for $\varepsilon$ does not duplicate the beta-binomial curve (Fig. 2A, long and short dashes, lowest curve). In fact, use of $n_{\text {deff }}$ consistently underestimates $p_{H i g h}$ almost as much as use of $n$ overestimates $p_{H i g h}$. An arbitrary 'temporary' value of $\varepsilon$ (selected from the range $n_{\text {deff }}$ to $n)$ was also used in this graph $(\varepsilon=5.2$; thick line), which pro-

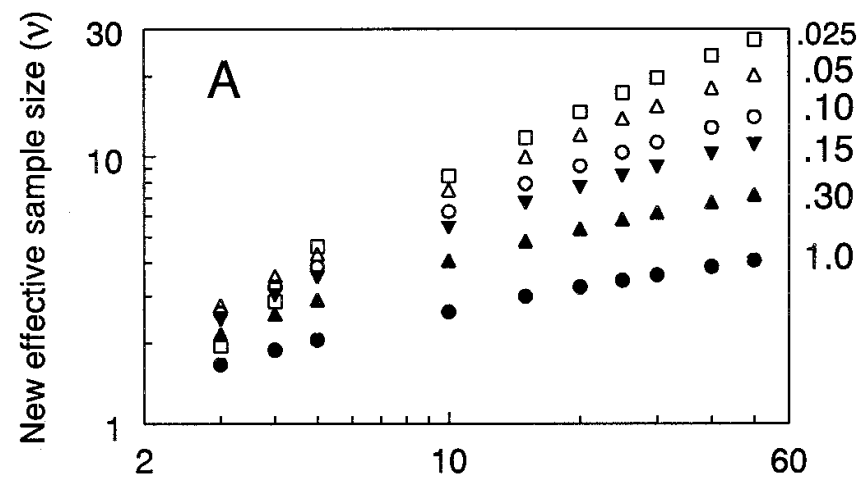

Sample size (n)
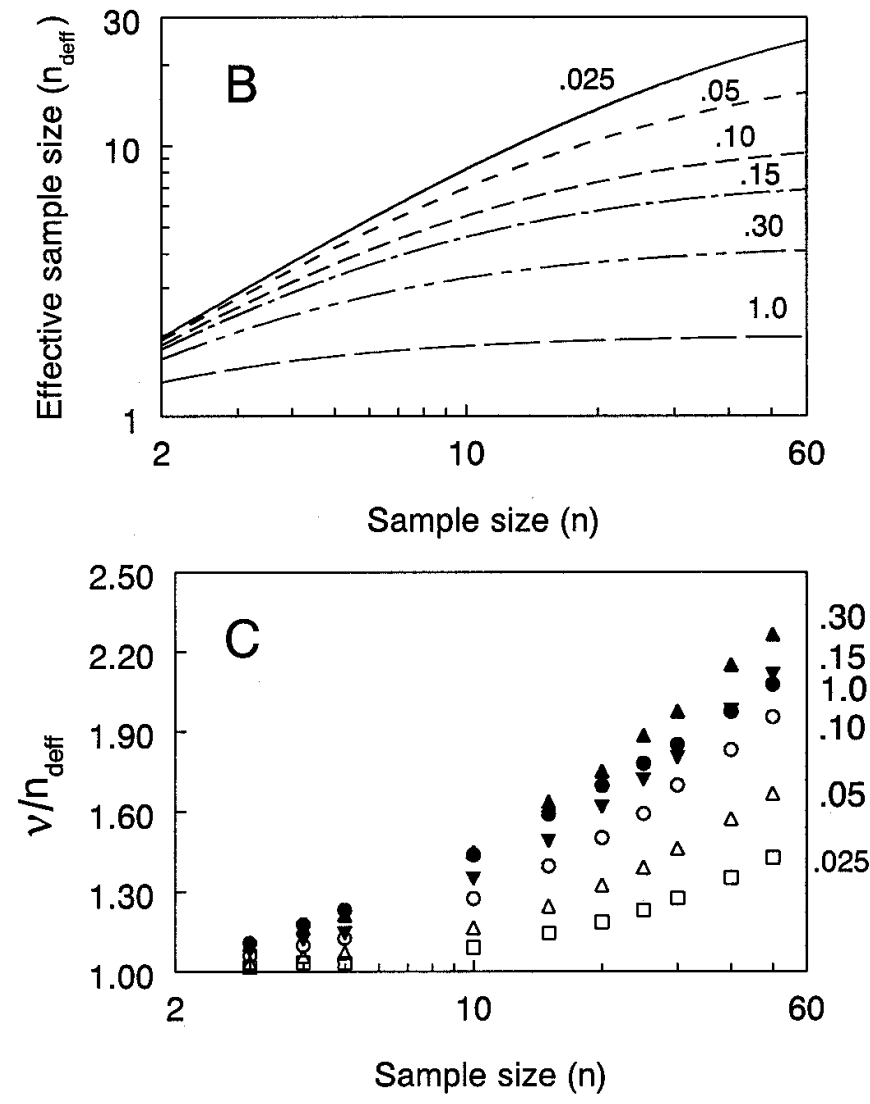

Fig. 4. A, New (or corrected or revised) effective sample size (v) for equating the zero terms of the beta-binomial and binomial distributions at selected values of the number of individuals per sampling unit $(n)$ and the index of aggregation of the beta-binomial distribution $(\theta)$. B, Effective sample size as defined by Kish (25) $\left(n_{\text {deff }}\right)$ in relationship to $n$ for selected values of $\theta$ (eq. 9 a). $\mathbf{C}, v / n_{\text {deff }}$ for selected $n$ and $\theta$ values. $\mathbf{A}$ and $\mathbf{C}$, Numbers to the right of the points and $\mathbf{B}$, next to the curves are the values of $\theta$. duced values of $p_{\text {High }}$ intermediate between the ones based on equation $1 \mathrm{~b}$ with $\varepsilon=n_{\text {deff }}$ and the ones based on equation 2 (with $n=$ 10 and $\theta=0.15$ ).

The effect of these different values of $\varepsilon$ can be seen more fully in plots of $\Delta p$ versus $p_{\text {Low }}$ (Fig. 2B). The difference between the binomial and beta-binomial curves (eqs. 6a and 7, respectively) was apparent, with the binomial curve considerably higher than the beta-binomial ones around $p_{\text {Low }}$ of 0.20 . The $n_{\text {deff }}$-based curve (eq. $6 \mathrm{~b}$ with $\varepsilon=n_{\text {deff }}$ ) was considerably below the beta-binomial one, and the maximum $\Delta p$ was at a larger $p_{\text {Low }}$ than for the beta-binomial-based curve. The curve based on the chosen (arbitrary) value of $\varepsilon$ (eq. $6 \mathrm{~b}$ with $\varepsilon=5.2$ ), intermediate between $n_{\text {deff }}$ and $n$, was closer to the beta-binomial curve than to either the binomial curve (based on eq. 1a) or the curve based on equation $1 \mathrm{~b}$ with $\varepsilon=n_{\text {deff. }}$.

The error in using the binomial formula was negative (Fig. 2C) because of the overprediction of $p_{\text {High }}$ (Fig. 2A); the greatest error, nearly -0.20 , was around $p_{\text {Low }}=0.13$. The error was positive with use of equation $1 \mathrm{~b}$ with $\varepsilon=n_{\text {deff }}$ (Fig. 2C) because of the underprediction of $p_{\text {High }}$ (Fig. 2A); maximum error was about 0.12. At high values of $p_{\text {Low }}$, the error was small for all cases, because $p_{\text {High }} \approx 1$ for equations 1a, 2, and $1 \mathrm{~b}$ (Fig. 2C).

Temporary $\varepsilon$ values were evaluated (for given $n$ and $\theta$ ) for their ability, when substituted into equation $1 \mathrm{~b}$, to produce $p_{\text {High }}$ values that came close to those produced by equation 2 based on the betabinomial distribution. The evaluation was based partly on visual inspection of plots using the format of Figure $2 \mathrm{~B}$ and $\mathrm{C}$ and partly on statistics calculated from the data presented in these plots. For each $\varepsilon$ value tested, the following statistics were determined: (i) mean absolute error (MAE), that is, the average of the absolute values of $p_{H i g h}$ from equation 2 (beta-binomial) minus $p_{\text {High }}$ from equation 1b; (ii) maximum absolute error (MAAE); and (iii) relative mean absolute error (RMAE), that is, MAE for a given value of $\varepsilon$ in equation $1 \mathrm{~b}$ divided by the MAE for the binomial function (eq. 1a). For RMAE, one can see the improvement in predictions of $p_{\text {High }}$ from equation $1 \mathrm{~b}$ compared with the prediction from the binomial equation 1a. The $\varepsilon$ values were chosen from the range $n_{\text {deff }} \leq \varepsilon \leq n$. Initial screening of $\varepsilon$ was done using the $\Delta p$ graph; $\varepsilon$ values were tested until the $\Delta p$ values of equation $6 \mathrm{~b}$ were about the same as the beta-binomial values (eq. 7) in the region of the curve in which $\Delta p$ was at the maximum. Integer and fractional values of $\varepsilon$, around the value found graphically, were then evaluated for error statistics and shape of the $\Delta p$ curve. For the example in Figure 2 and temporary value of $\varepsilon=5.2$, MAE $=0.021$, MAAE $=0.068$, and RMAE $=0.424(42.4 \%)$.

The value of $\varepsilon$ that resulted in the smallest MAE was chosen as the best $\varepsilon$. We call this value $v$ (Greek 'nu'). The new equation is written as

$$
p_{\text {High }}=1-\left(1-p_{\text {Low }}\right)^{v}
$$

With $n=10$ and $\theta=0.15, v=6.2$. Error statistics were MAE $=$ 0.003 , MAAE $=0.007$, and RMAE $=0.064(6.4 \%)$. Results can be seen in Figure 3, which is the same format as Figure 2, with the curves corresponding to the temporary $\varepsilon=5.2$ replaced with curves for $v=6.2$. The agreement is so good here that in Figure $3 \mathrm{~A}$ and $\mathrm{B}$ one cannot see separate curves for the beta-binomial-based function (eq. 2) and equation $1 \mathrm{c}$ (eq. $1 \mathrm{~b}$ with $\varepsilon=v$ ). Maximum value of $\Delta p$ was 0.59 for both the beta-binomial and approximation curves (Fig. 3B) The error (Fig. 3C) was generally very small for all values of $p_{\text {Low }}$; the largest errors were positive (predicted $p_{\text {High }}$ was less than $p_{\text {High }}$ of the beta-binomial distribution), and these were for values of $p_{\text {Low }}$ around 0.1 (Fig. 3C).

Testing of $\boldsymbol{n}$ and $\boldsymbol{\theta}$ values. The procedure outlined above was repeated for $n=3,4,5,10,15,20,25,30,40$, and 50 and for $\theta=$ $0.025,0.05,0.10,0.15,0.30$, and 1.0 for each $n$. All the values of $\theta$ are typical, based on published studies $(16,27)$, except for $\theta=$ 1.0. This large value was chosen to obtain an extreme (but not impossible [Fig. 5H in literature citation 27]) situation for testing the approximation method. The chosen $v$ values generally met the fol- 
lowing criteria: MAE $<0.01$, MAAE $<0.025$, and RMAE $<0.15$. In addition to producing low MAE and related error statistics, use of $v$ produced a $\Delta p$ curve that was qualitatively similar to the $\Delta p$ curve for the beta-binomial (Fig. 3B). The largest MAEs were for $\theta=1$, in which the approximate nature of equation $1 \mathrm{c}$ was discernible.

The selected $v$ values increased with $n$ (Fig. 4A), similar to the manner in which $n_{\text {deff }}$ increases with $n$ (Fig. 4B). At large $n(n=$ 25 to 50), the rate of increase in $v$ with increasing $n$ was greater than the rate of increase in $n_{\text {deff }}$ with $n$. The values of $v$ in Figure 4A are shown as points rather than curves to emphasize that the selection procedure involved individual $n$ and $\theta$ values rather than all possible values of these two terms. On the other hand, $n_{\text {deff }}$ is shown as a curve (Fig. 4B) because of the exact functional relationship between $n_{\text {deff }}$ and $n$ (eq. 9a).

The values of $v$ decreased with increasing $\theta$ at all levels of $n$ tested (Fig. 4A). This is qualitatively similar to the change in $n_{\text {deff }}$ with $\theta$ (Fig. 4B). However, $v$ was greater than the corresponding $n_{\text {deff }}$ for any given combination of $n$ and $\theta$ tested. This can be seen from the ratio $v / n_{\text {deff }}$ in relation to $n$ and $\theta$ (Fig. $4 \mathrm{C}$ ). This ratio ranged from $\sim 1.05$ to $\sim 2.25$ under the conditions tested here and increased with $n$. At $n<5$, the ratio was only slightly greater than 1 , especially at low $\theta$, but at $n>25$, the ratio could be greater than 2.0. Interestingly, the ratio increased with $\theta$ for $\theta \leq 0.30$, and then the ratio declined for the very large $\theta$ (Fig. 4C).

An empirical equation was developed to predict $v$ based on $n$ and $\theta$, using ordinary least squares regression. The selected equation was

$$
\hat{v}=\frac{n}{0.98135+0.8179 \theta+0.004958 n+0.30387 \theta n-0.3471 \theta^{2}-0.08475 \theta^{2} n}
$$

which had a coefficient of determination $\left(R^{2}\right)$ of 0.998 (for the values of $n$ and $\theta$ listed above). This equation is not meant to represent an exact mathematical relationship between $v$ and $n$ and $\theta$, but rather an approximation to use for selecting $v$ when not using the Mathcad (or equivalent) routine or for choosing the first guess of $v$ when using the iterative method. For the example in Figure 3, $\hat{v}=6.3$. Equation 10 is the same format as the one for $n_{\text {deff, that }}$ is, $n$ divided by a function of $n$ and $\theta$ (eq. 9a).

Comparison with an exact result. Although use of equation 1c can produce values of $p_{\text {High }}$ very close to those specified by the beta-binomial distribution (eq. 2), the agreement is not perfect at any value of $n$ or $\theta$. That is, MAE and MAAE were never 0 for the tested conditions in Figure 4, even though these error statistics were small and the curves for the beta-binomial and the approximation were visually indistinguishable (Fig. 3) in many cases.

The relationship is only an approximation, because an equation based on the zero term of the binomial distribution (with the same general format as eq. 1b or 1c) can only exactly duplicate equation 2 (based on the zero term of the beta-binomial distribution) if the exponent is a variable. Denoting this variable $E$, we can write

$$
p_{\text {High }}=1-\left(1-p_{\text {Low }}\right)^{E}
$$

After equating equations $1 \mathrm{~d}$ and 2 , algebraic rearrangement results in

$$
E=\frac{\sum_{i=0}^{n-1} \ln \frac{1-p_{\text {Low }}+i \theta}{1+i \theta}}{\ln \left(1-p_{\text {Low }}\right)}
$$

$E$ is, therefore, a function of $\theta$ and $p_{\text {Low. }}$. Plotting $E$ versus $p_{\text {Low }}$ at selected $n$ and $\theta$ shows how $E$ varies with $p_{\text {Low }}$ (Fig. 5A and B). $E$ is greater than $n_{\text {deff }}$ and changes only slightly with $p_{\text {Low }}$ for values of $p_{\text {Low }} \lesssim 0.50$. At higher $p_{\text {Low }}$, the change in $E$ with change in incidence is greater, and at $p_{\text {Low }} \approx 0.8, E$ crosses below the horizontal $n_{\text {deff }}$ line. Also shown in Figure 5A and B is the value of $v$ (according to the criteria given above). The $v$ values correspond to $E$ at a $p_{\text {Low }}$ of about 0.20 . This does not correspond to any obvious critical level of $p_{\text {Low }}$; for instance, this $p_{\text {Low }}$ is not the value in which $\Delta p$ is a maximum (Fig. 3B).
Although equation 11 shows that one cannot exactly duplicate the beta-binomial zero term with a binomial function and a constant exponent, one can come very close to duplicating this by careful selection of a constant $(v)$. This $v$ value corresponds roughly to the region of the $E: p_{\text {Low }}$ curve in which there is little change in $E$ with change in $p_{\text {Low }}$. If one were interested in predicting $p_{\text {High }}$ of the beta-binomial only for large $p_{\text {Low }}$, then one might, at first, consider use of an $\varepsilon$ (eq. $1 \mathrm{~b}$ ) equal to $n_{\text {deff }}$ rather than equal to $v$ (eq. $1 \mathrm{c}$ ), because $E$ is close to $n_{\text {deff }}$ at fairly high $p_{\text {Low }}$ (Fig. 5A). However,
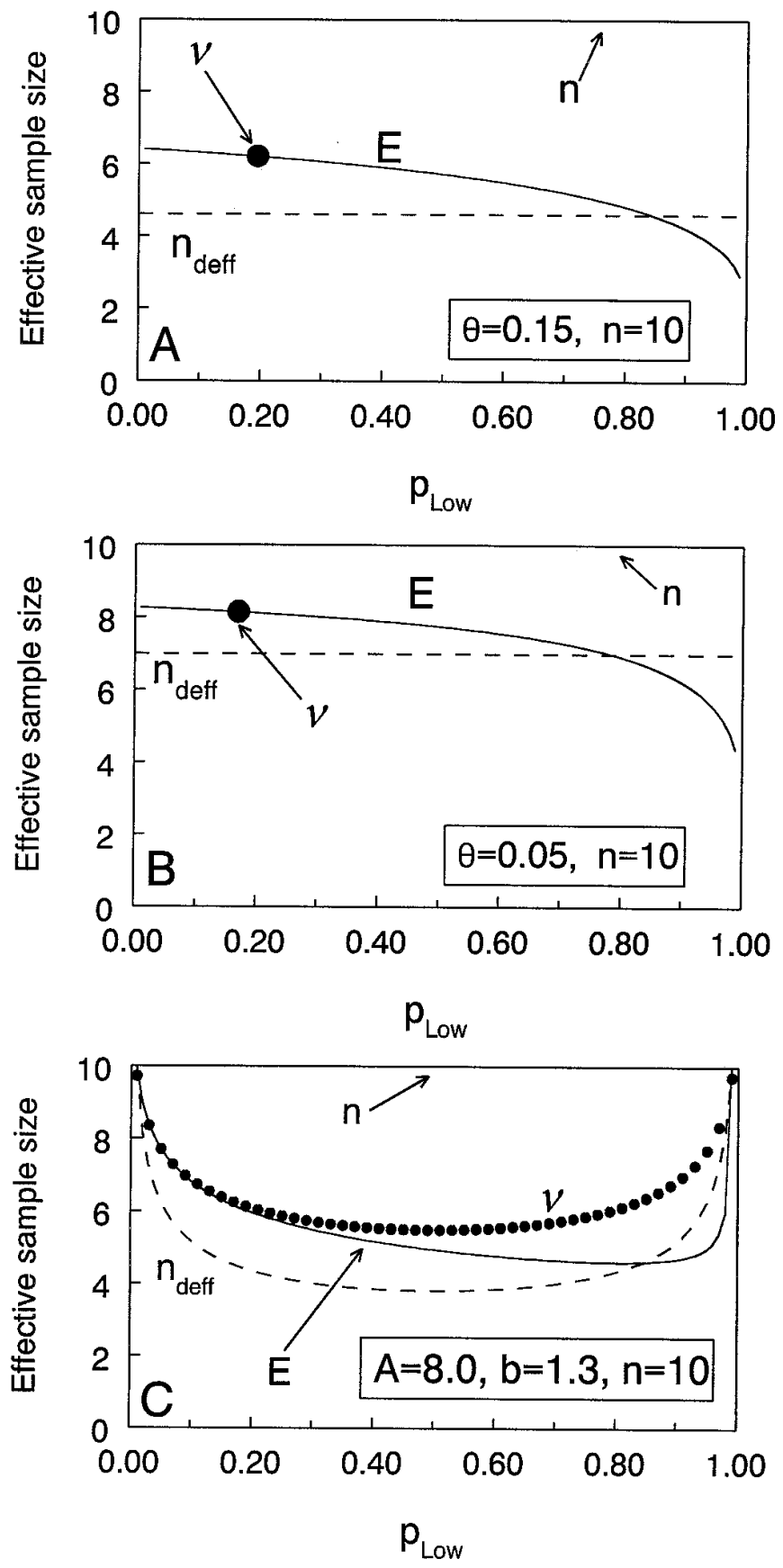

Fig. 5. Types of effective samples sizes that can be used to represent (approximately or exactly) aspects of the beta-binomial distribution with the binomial distribution for number of individuals per sampling unit $(n)$ equal to 10 and for index of aggregation $(\theta)$ being fixed or variable. Graphs show: Kish's (25) effective sample size $\left(n_{\text {deff }}\right)$ (eq. 9a), new effective sample size $(v)$ for approximately the zero terms of the beta-binomial and binomial distributions, and the variable effective sample size $(E)$ for exactly duplicating the zero term of the beta-binomial with the binomial distribution (eq. 11). A, $\theta=0.15$. $\mathbf{B}, \theta=0.05$. C, $\theta$ varies according to the binary power law (15) with parameters $A$ and $b$ shown on the graph; $\theta$ is predicted with equation 12 . $\mathbf{A}$ and $\mathbf{B}, v$ is chosen with the iterative method; $\mathbf{C}$, equation 10 is used to predict $v$. 
the values of $p_{H i g h}$ corresponding to $p_{\text {Low }}$ are not presented in Figure 5. At relatively large $p_{\text {Low }}, p_{\text {High }}$ for the beta-binomial distribution (eq. 2) is 1 or almost 1 for realistic values of $\theta$ (Fig. 1A), and a relatively wide range of possible $\varepsilon$ s would give almost the same predicted $p_{\text {High }}(\sim 1)$. Thus, there is little advantage in focusing on the larger values for $p_{\text {Low }}$ under most circumstances. The advantage of focusing on small values of $p_{\text {Low }}$ in selecting $v$ is that, here, $p_{\text {High }}$ can vary the most with different values of $n$ and $\theta$. It is also the case that small values of $p_{\text {Low }}$ are normally the ones of greatest interest in the context of disease management decision making.

Nonconstant aggregation of disease incidence. In many pathosystems, degree of aggregation of incidence at the lower level in a spatial hierarchy, as measured by $\theta$, varies with $p_{\text {Low }}(15,22$, $27,28)$. This variable aggregation can typically be represented by the binary power law in which the logarithm of the observed variance of incidence is a linear function of the theoretical variance for a binomial distribution. One way of writing the binary power law $(15,28,30)$ is $s^{2}=A \cdot\left(s^{2}{ }_{\text {in }}\right)^{b}$, in which $s^{2}$ is the calculated observed variance of the proportions per sampling unit (calculated variance of $\left.X_{j} / n, j=1, \ldots, N\right), s_{b i n}^{2}$ is the calculated theoretical variance for a binomial distribution $\left(\hat{p}_{\text {Low }}\left[1-\hat{p}_{\text {Low }}\right] / n\right)$, and $A$ and $b$ are parameters. In this formulation, one can show that $\theta$ is equal to a function of $p_{\text {Low }}, A$, and $b(27)$

$$
\theta=\frac{A n^{-b}-\left[\hat{p}_{\text {Low }}\left(1-\hat{p}_{\text {Low }}\right)\right]^{1-b} / n}{\left[\hat{p}_{\text {Low }}\left(1-\hat{p}_{\text {Low }}\right)\right]^{1-b}-A n^{-b}}
$$

When $b>1, \theta$ is small at small $p_{\text {Low }}$, rises to a maximum at $p_{\text {Low }}=$ 0.5 , and then declines to small values. When $b=1$, equation 12 reduces to a constant, $\theta=(A-1) /(n-A)$, with $A$ being then interpreted as a fixed $\operatorname{deff}$ (eq. 8).

As done by Hughes et al. (21), the variability in $\theta$ can be directly taken into account for predicting $p_{\text {High }}$ from $p_{\text {Low }}$ by substituting equation 12 for $\theta$ into equation 2 . An equivalent method can be followed for the situation in which the beta-binomial expression (right-hand side of eq. 2) is approximated by a binomial-type expression (right-hand side of eq. 1c). One would simply use equation 12 to predict $\theta$ over the range of $p_{\text {Low }}$ values, estimate the corresponding $v$ s with equation 10 , and then use these $v$ values in equation 1c. For the special case of Kish's effective sample size (25), it can be shown that one can write $n_{\text {deff }}$ as

$$
n_{\text {deff }}=n^{b}\left[\hat{p}_{\text {Low }}\left(1-\hat{p}_{\text {Low }}\right)\right]^{1-b} / A
$$

because there is an exact mathematical relationship between $\theta$ and deff (eq. 8). Finally, variability of $\theta$ could be incorporated into the exact representation of the beta-binomial zero term by a binomialtype function (eq. 1d). Specifically, one predicts $\theta$ over the range of $p_{\text {Low }}$ values using equation 12 and substitutes these predicted $\theta \mathrm{s}$ into equation 11 to determine the corresponding values of $E$. Values of $p_{H i g h}$ can then be obtained from equation $1 \mathrm{~d}$.

The influence of $\theta$ (eq. 12) on $n_{\text {deff, }}, \mathrm{v}$, and $E$ is shown in Figure 5C for $A=8.0$ and $b=1.3$. These are typical values for the binary power law parameters $(15,27)$. Predicted $\theta$ for this situation is $\sim 0.0$ at $p_{\text {Low }}=0.01$, rising to 0.22 at $p_{\text {Low }}=0.50$, and then declining to $\sim 0.0$ at $p_{\text {Low }}=0.99$ (based on eq. 12). Mean $\theta$ over the range of 0.01 to 0.99 incidence is 0.16 , similar to the fixed $\theta$ of 0.15 in Figure 5A. As specified by equation $9 \mathrm{~b}, n_{\text {deff }}$ starts high (near $n$ ) at small $p_{\text {Low }}$, declines to about 4 for incidences from 0.2 to 0.8 , and then rises again at large $p_{\text {Low }}$ (Fig. $5 \mathrm{C}$ ). The exact exponent $(E)$ is larger than $n_{\text {deff }}$ for $p_{\text {Low }} \lesssim 0.80$, as found for fixed $\theta$ (Fig. 5A). The $E$ and $n_{\text {deff }}$ curves cross at about an incidence of 0.8 , and then both increase because of the decreasing $\theta$ at large $p_{\text {Low }}$.

The predicted values of $v$ (based on eq. 10, with $\theta$ predicted from eq. 12) are also larger than $n_{\text {deff }}$ values (Fig. 5C) but follow the same pattern in relation to $p_{\text {Low }}$. The $v$ values are very similar to the $E$ values at $p_{\text {Low }} \lesssim 0.4$, as also found for fixed $\theta$ (Fig. 5A). The major discrepancy between $v$ and $E$ occurs at relatively large $p_{\text {Low }}$, which would correspond to very large $p_{\text {High }}$; this is a situation in which a range of values between $v$ and $E$ (or between $n$ and $n_{\text {deff }}$ ) would give nearly the same $p_{\text {High }}$.

\section{MATERIALS AND METHODS}

The ability to use equation $1 \mathrm{c}$ to predict disease incidence at one level in a spatial hierarchy from incidence at another level was evaluated using (i) field observations of diseased grapevines and (ii) beta-binomial simulations of diseased individuals.

Field data. The spatial pattern of Eutypa dieback of grape (caused by Eutypa lata) in California was previously characterized using several methods $(20,29,34)$. There were 22 data sets, consisting of observations of diseased vines in vineyards over 3 years. Vineyards consisted of $N=144$ to 372 sampling units (quadrats),

TABLE 1. Summary statistics of simulations ${ }^{\mathrm{a}}$ for the error in predicting disease incidence at the lower level in a spatial hierarchy (e.g., individual leaves) based

\begin{tabular}{|c|c|c|c|c|c|c|c|}
\hline \multicolumn{2}{|c|}{ Simulation condition $^{\mathrm{b}}$} & \multirow[b]{2}{*}{ Error statistic ${ }^{\mathrm{c}}$} & \multicolumn{5}{|c|}{ Prediction method $^{\mathrm{d}}$} \\
\hline$\theta$ & $p_{\text {Low }}$ & & $n$ (eq. 13a) & $n_{\text {deff }}($ eq. 13b) & $v($ eq. $13 \mathrm{c})$ & $v(\theta \times 1.5)($ eq. $13 \mathrm{~d})$ & $v(\theta \div 1.5)($ eq. $13 \mathrm{e})$ \\
\hline \multirow[t]{3}{*}{0.05} & 0.20 & ME: & 0.028 & -0.036 & -0.0066 & -0.022 & 0.005 \\
\hline & & MAE: & 0.031 & 0.038 & 0.022 & 0.028 & 0.021 \\
\hline & & $Q_{3}:$ & 0.047 & 0.055 & 0.032 & 0.041 & 0.031 \\
\hline \multirow[t]{3}{*}{0.15} & 0.20 & ME: & 0.069 & -0.063 & -0.0027 & -0.033 & 0.020 \\
\hline & & MAE: & 0.069 & 0.063 & 0.022 & 0.036 & 0.027 \\
\hline & & $Q_{3}:$ & 0.083 & 0.083 & 0.030 & 0.052 & 0.040 \\
\hline \multirow[t]{3}{*}{0.45} & 0.20 & ME: & 0.114 & -0.087 & -0.005 & -0.044 & 0.026 \\
\hline & & MAE: & 0.114 & 0.087 & 0.020 & 0.045 & 0.029 \\
\hline & & $Q_{3}$ & 0.127 & 0.108 & 0.028 & 0.063 & 0.040 \\
\hline \multirow[t]{3}{*}{0.15} & 0.05 & ME: & 0.019 & -0.018 & -0.003 & -0.009 & 0.006 \\
\hline & & MAE: & 0.019 & 0.019 & 0.008 & 0.012 & 0.009 \\
\hline & & $Q_{3}$ & 0.026 & 0.025 & 0.011 & 0.016 & 0.013 \\
\hline \multirow[t]{3}{*}{0.15} & 0.40 & ME: & 0.086 & -0.118 & -0.030 & -0.075 & 0.006 \\
\hline & & MAE: & 0.150 & 0.101 & 0.059 & 0.067 & 0.074 \\
\hline & & $Q_{3}$ & 0.173 & 0.138 & 0.072 & 0.092 & 0.097 \\
\hline
\end{tabular}
on the calculated disease incidence at the higher level (e.g., sampling unit)

a There were 200 replicate simulations of $N=50$ sampling units and $n=10$ individuals per sampling unit.

b Data were generated to follow a beta-binomial distribution with parameters $p_{\text {Low }}$ and $\theta$.

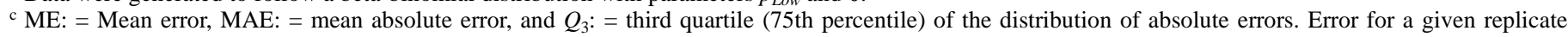
simulation is the actual proportion of diseased individuals ( $\hat{p}_{\text {Low }}$ ) minus the proportion predicted by equations 13 a to $13 \mathrm{e}\left(\tilde{p}_{\text {Low }}\right)$ based on $\hat{p}_{\text {High }}$.

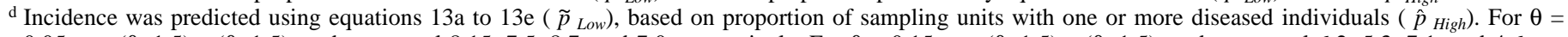
$0.05, v, v(\theta \times 1.5), v(\theta \div 1.5)$, and $n_{\text {deff }}$ equal 8.15, 7.5, 8.7, and 7.0, respectively. For $\theta=0.15, v, v(\theta \times 1.5), v(\theta \div 1.5)$, and $n_{\text {deff }}$ equal $6.2,5.3,7.1$, and 4.6, respectively. For $\theta=0.45, v, v(\theta \times 1.5), v(\theta \div 1.5)$, and $n_{\text {deff }}$ equal 3.9, 3.2, 4.7, and 2.64, respectively. 
each consisting of $n=9$ vines. The beta-binomial distribution was previously found to provide a good fit to the data in the individual data sets (29) for diseased vines, and the binary power law (15) provided a precise description of the variances across the 22 data sets. Estimated $b$ was not significantly different from 1 ; thus, there was no evidence that degree of aggregation (as measured by $\theta$ ) varied systematically with $p_{\text {Low }}$. The maximum likelihood estimate of a single $\theta$ for the collection of data sets was 0.053 (29).

Although there has been considerable analysis of these Eutypa data, the $p_{\text {High }}: p_{\text {Low }}$ relationship had not been previously assessed. The ability to predict $\hat{p}_{\text {High }}$ from $\hat{p}_{\text {Low }}$ (to obtain $\widetilde{p}_{\text {High }}$ ) was, thus, evaluated here based on use of equations 1a (binomial-based function), 2 (beta-binomial), $1 \mathrm{~b}$ (binomial with $\varepsilon=n_{\text {deff }}$ replacing $n$ ), and 1c (binomial with $v$ replacing $n$ ). Selection of $v$ was based on the values of $n$ and estimated $\theta$ for diseased vines, as discussed above. Predictions were assessed based on the calculated MAE for the difference between $\hat{p}_{\text {High }}$ and $\widetilde{p}_{\text {High }}$.

Simulation. A Monte Carlo simulation was performed in order to evaluate the method of using a binomial-type formula for predicting disease incidence at the lower scale $\left(\widetilde{p}_{\text {Low }}\right)$ based on observations at the higher scale $\left(\hat{p}_{\text {High }}\right)$. As with the other analyses discussed above, the approach here was based on the assumption that the beta-binomial distribution describes the disease incidence data at the lower level.

A beta-binomial random sample was simulated using the method described in Ahn and Chen (1). Briefly, a Mathcad worksheet was written to generate, one at time, a value $(\pi)$ from a beta probability density function (with parameters $p_{\text {Low }} / \theta$ and $\left[1-p_{\text {Low }}\right] / \theta$ ). A binomial variate was then generated with parameters $\pi$ and $n$. This was repeated for a total of $N$ times for $N$ sampling units. Mathcad's standard statistical distribution generator was used for simulating the data.

For selected values of $p_{\text {Low }}$ and $\theta$ (discussed below), the simulations consisted of 200 replications $(k=1, \ldots, 200)$ of $N=50$ sampling units, comprising $n=10$ individuals in each sampling unit. Thus, the number of diseased individuals in the $j$ th sampling unit of the $k$ th replication $\left(X_{j, k}\right)$ ranged from 0 to $10(=n)$. For each of the 200 replications, the moment estimates of $p_{\text {Low }}$ and $\theta$ ( $\hat{p}_{\text {Low }, k}$ and $\hat{\theta}_{k}$ ) were calculated based on the proportion of the $n \cdot N$ individuals diseased and equation 3 , respectively. The moment estimate of $p_{\text {High }}$ for the individual replications $\left(\hat{p}_{H i g h, k}\right)$ based on the proportion of the 50 sampling units with any diseased individuals also was calculated. The means of $\hat{p}_{\text {Low }, k}$ and $\hat{\theta}_{k}$ across the 200 replications were determined to confirm that the simulations were resulting in the appropriate (desired) values of $p_{\text {Low }}$ and $\theta$.

Simulations were performed for the following combinations of incidence and degree of aggregation $\left(p_{\text {Low }}, \theta\right)$ : $(0.20,0.05),(0.20$, $0.15),(0.20,0.45),(0.05,0.15)$, and $(0.40,0.15)$. Means of estimated incidence and aggregation at the lower level across the replications $\left(\bar{p}_{\text {Low }}, \bar{\theta}\right)$ were $(0.201,0.051),(0.200,0.152),(0.199$, $0.470),(0.051,0.159)$, and $(0.399,0.150)$. This indicates that betabinomial variables with the desired properties were obtained with the simulation method. For each $p_{\text {Low }}$ and $\theta$, deff (eq. 8) and $n_{\text {deff }}$ (eq. 9a) were determined. Using the Mathcad procedure discussed above, $v$ was also selected for each chosen $\theta$ before the simulations were performed. For $\theta=0.05, n_{\text {deff }}=7.0$ and $v=8.15$; for $\theta=$ $0.15, n_{\text {deff }}=4.6$ and $v=6.2$; for $\theta=0.45, n_{\text {deff }}=2.64$ and $v=3.9$.

Using the $\hat{p}_{H i g h, k}$ values calculated as indicated above, incidence at the lower level was predicted with the binomial-based formula (eq. 1a) rearranged as in equation 4 and with similar rearrangements of equation $1 \mathrm{~b}$ with $\varepsilon=n_{\text {deff }}$ and with equation $1 \mathrm{c}$. Specifically, with the parameters replaced by their estimates, these equations can be written for individual replications as

$$
\begin{gathered}
\tilde{p}_{\text {Low }, k}=1-\left(1-\hat{p}_{H i g h, k}\right)^{1 / n} \\
\tilde{p}_{\text {Low }, k}=1-\left(1-\hat{p}_{H i g h, k}\right)^{1 / \text { deff }}
\end{gathered}
$$

and

$$
\tilde{p}_{\text {Low }, k}=1-\left(1-\hat{p}_{H i g h, k}\right)^{1 / v}
$$

Use of equations $13 \mathrm{~b}$ and $13 \mathrm{c}$ require knowledge of $\theta$; although this is not a difficulty with the simulation; in practice, $\theta$ will not be exactly known if only $\hat{p}_{\text {High }}$ is determined. Thus, two variations of equation $13 \mathrm{c}$ were tested in which the assumed $\theta$ is incorrect: (i) in which the 'guessed' value of $\theta$ is $50 \%$ larger than the actual value $(\theta \times 1.5)$ and (ii) in which the 'guessed' value of $\theta$ is two-thirds of the actual value $(\theta \div 1.5)$, that is, in which the actual $\theta$ is $50 \%$ larger than the 'guessed' value. For example, for the simulation with true $\theta=0.15$, vs corresponding to $\theta$ s of 0.225 $(\theta \times 1.5)$ and $0.10(\theta \div 1.5)$ were selected, in addition to $v$ for $\theta=$ 0.15 . We label these new $v$ values as $v(\theta \times 1.5)$ and $v(\theta \div 1.5)$. The two new equations can be written as

$$
\tilde{p}_{\text {Low }, k}=1-\left(1-\hat{p}_{H i g h, k}\right)^{1 / v(\theta \times 1.5)}
$$

and

$$
\tilde{p}_{\text {Low }, k}=1-\left(1-\hat{p}_{H i g h, k}\right)^{1 / v(\theta \div 1.5)}
$$

Values of $v(\theta \times 1.5)$ and $(\theta \div 1.5)$ are given in the footnotes of Table 1. In total, five equations (eqs. 13a to 13e) were used to provide predictions of $p_{\text {Low }}$.

Although one cannot rearrange the beta-binomial equation 2 in the same manner as done for the binomial-type functions, for the simulations, information is available on the actual estimates of
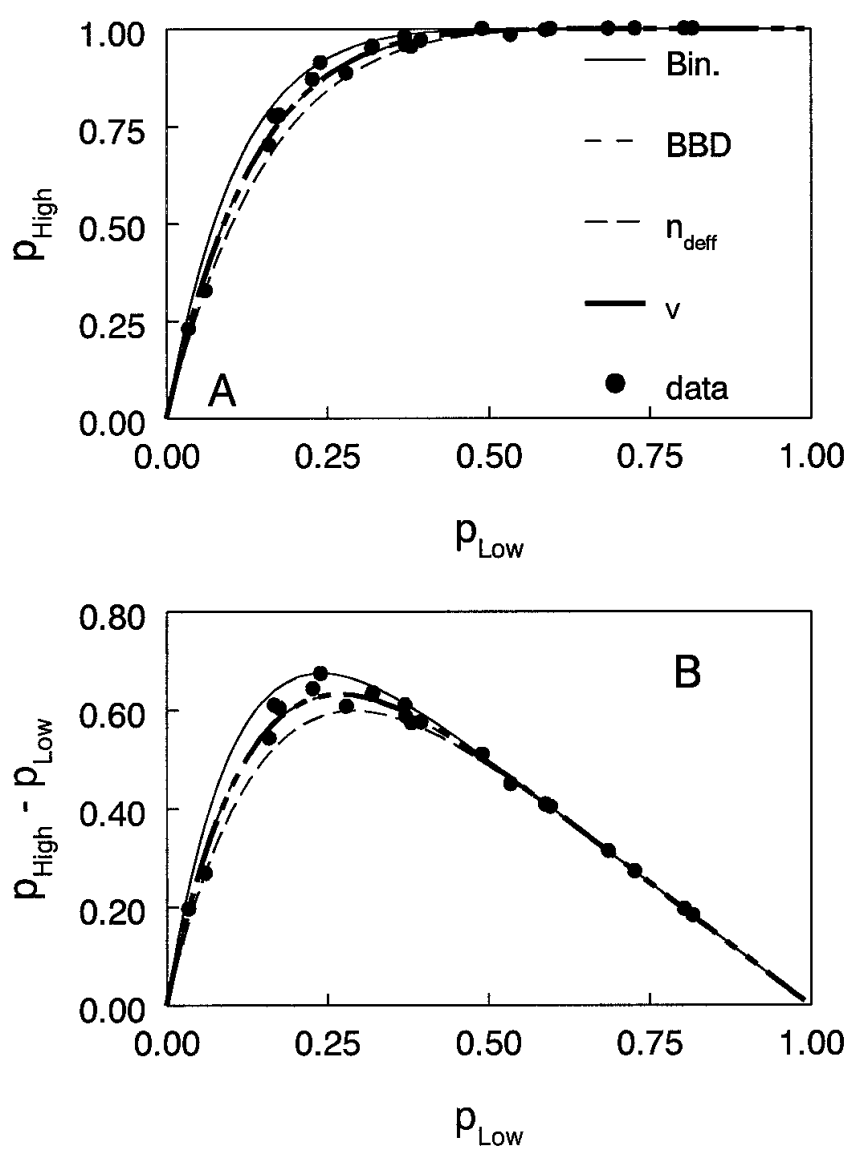

Fig. 6. Relationship between incidence of Eutypa dieback of grape vines (34) at two levels in a spatial hierarchy, together with predictions based on various models. A, Estimated expected probability of vines being diseased ( $\left.\hat{p}_{\text {Low }}\right)$ in relation to the estimated probability of a sampling unit (quadrat with $n=9$ vines) having one or more diseased individuals $\left(\hat{p}_{\text {High }}\right)$ ('• data'), together with the predictions based on the beta-binomial distribution (eq. 2) with $\hat{\theta}=$ 0.053 ('BBD'); equation 1a for the binomial distribution ('Bin.'); equation $1 \mathrm{~b}$ with $\varepsilon=n_{\text {deff }}=7.14$ (' $n_{\text {deff }}$ ); and equation $1 \mathrm{c}$ with $v=7.4$ (' $v$ '). B, Relation between $\hat{p}_{\text {Low }}$ and the difference between $\hat{p}_{\text {High }}$ and $\hat{p}_{\text {Low }}(\Delta p)$ in $\mathbf{A}$. 
$p_{\text {Low }}$ for each replication $\left(\hat{p}_{\text {Low }, k}\right)$. These $\hat{p}_{\text {Low }, k}$ values are considered here as the true values. For each replication, the error $(\xi)$ in prediction of $p_{\text {Low }}$ based on $p_{\text {High }}$ was determined as

$$
\xi_{k}=\hat{p}_{\text {Low }, k}-\tilde{p}_{\text {Low }, k}
$$

and various summary statistics were calculated including mean error (ME); MAE; and third quartile (75th percentile) of the distribution of absolute errors $\left(Q_{3}\right)$. Twenty-five percent of the absolute errors are greater than or equal to $Q_{3}$. ME is a measure of the accuracy of the predictions, giving a 0 if predictions based on the higher scale are unbiased (even if there is high variability around the true values). MAE and $Q_{3}$ both measure the precision and accuracy of the predictions.

\section{RESULTS}

Field data. Based on the estimated $\theta(=0.053)$ for the Eutypa dieback data sets (29), an estimated deff of 1.4 (eq. 8) was obtained, which resulted in an $n_{\text {deff }}$ of 7.14 (=10/1.4 [eq. 9a]). The direct estimates of $p_{\text {Low }}$ from the individual vine data $\left(\hat{p}_{\text {Low }}\right)$ ranged from 0.032 to 0.817 ; direct estimates of $p_{H i g h}$, based on the proportion of sampling units with diseased vines $\left(\hat{p}_{\text {High }}\right)$, ranged from 0.20 to 1.0. A plot of $\hat{p}_{\text {High }}$ versus $\hat{p}_{\text {Low }}$ for Eutypa dieback showed the standard saturation-type relationship (Fig. 6A), with several $\hat{p}_{H i g h} \mathrm{~s}$ close to or equal to 1.0. As expected, based on the consistent degree of overdispersion across these data sets (binary power law $b$ equal to 1 [29]), the predicted $p_{H i g h}$ s based on the binomialbased formula $\left(\tilde{p}_{\text {High }}\right)$ (eq. 1a with $\hat{p}_{\text {Low }}$ for $p_{\text {Low }}$ ) were larger than the observed $\hat{p}_{H i g h} \mathrm{~s}$, except, of course, when $\hat{p}_{\text {High }}=1$. The differences between the $\hat{p}_{H i g h} \mathrm{~S}$ and binomial predictions were not large (Fig. 1A) because estimated $\theta$ was relatively low $(\hat{\theta}=0.053)$. MAE for the difference between $\hat{p}_{\text {High }}$ and $\tilde{p}_{\text {High }}$ (not for the difference between the beta-binomial and the binomial predictions, as done for $v$ selections) was 0.024 . The predictions of $p_{\text {High }}$ from the beta-binomial-based equation 2 passed through the collection of observed data points (Fig. 6A), and MAE $=0.014$, about half the value obtained with the binomial predictions. It should be noted that there was no curve fitting involved in determining this beta-binomial curve (or any of the other curves in Fig. 6A); that is, the curve through the data points was entirely determined by the quantified level of aggregation of disease incidence at the lower level in the hierarchy.

Predicted $p_{\text {High }} \mathrm{s}$ based on equation $1 \mathrm{~b}$ with $\varepsilon=n_{\text {deff }}$ were generally lower than the $\hat{p}_{H i g h} \mathrm{~s}$ (Fig. 6A). MAE $=0.024$, the same as MAE for the binomial-based function (eq. 1a). In a sense, in this example, the predictions based on $n$ and $n_{\text {deff }}$ mostly set bounds around $\hat{p}_{\text {High }}$, with the beta-binomial predictions (eq. 2) falling between these bounds. Of course, these bounds are not viewed here in a probabilistic sense.

Using the methodology discussed in this paper, $v$ was found to equal 7.4 for $n=9$ and $\hat{\theta}=0.053$. Equation 1c then resulted in predicted $p_{H i g h} \mathrm{~s}\left(\tilde{p}_{H i g h} \mathrm{~s}\right)$ that were almost identical to those determined with the beta-binomial-based function (eq. 2). The agreement was so good that one cannot see the separate curves for equation 2 and equation 1c in Figure 6A. The MAE for the agreement of $\widetilde{p}_{\text {High }}$ values based on equation 1c $(v=7.4)$ with the observed data was 0.013, showing agreement with the data that was at least as good as that achieved with the direct use of the beta-binomialbased (eq. 2) formula.

With the $\Delta p$ plot (Fig. 6B), one can see the influence of using $n$ or $n_{\text {deff }}$ on predictions of $\Delta p$ (and, thus, $\widetilde{p}_{\text {High }}$ ) at intermediate $\hat{p}_{\text {Low }}(\sim 0.25)$. At $\hat{p}_{\text {Low }}=0.25, \Delta p$ based on the binomial function (eq. 6a) was 0.67 , and $\Delta p$ based on equation $6 \mathrm{~b}$ with $\varepsilon=n_{\text {deff }}$ was 0.59. Most of the observations were between these extremes in this region of $\hat{p}_{\text {Low }}$. $\Delta p$ based on the beta-binomial-based function (eq. 7) and equation $6 \mathrm{~b}$ with $\varepsilon=v$ overlapped, and both curves passed through the data points. At $\hat{p}_{\text {Low }} \gtrsim 0.5$, the $\Delta p$ s were not dependent on the form of the prediction equations because $\hat{p}_{\text {High }}=$ 1 , or nearly 1 , in this range of $\hat{p}_{\text {Low }}$.

Simulation. Predictions of disease incidence at the lower level ( $\tilde{p}_{\text {Low }}$ ) using the standard binomial-based formula (eq. 13a) were lower than the true incidence values $\left(\hat{p}_{\text {Low }}\right)$ for all tested Monte Carlo simulation conditions of $\theta$ and $p_{\text {Low }}$ (Table 1, positive ME). Furthermore, predictions of disease incidence using $n_{\text {deff }}$ (eq. 13b) overestimated the true values for all conditions (Table 1, negative $\mathrm{ME}$ ). Use of $v$ (eq. 13c) produced the most accurate and precise estimates of disease incidence. Over- or underestimating $\theta$ (and, hence under- or overestimating $v$, respectively [eqs. 13d and 13e]) decreased the accuracy and precision of the predictions compared with use of the exact $\theta$ (eq. 13c), but the $\widetilde{p}_{\text {Low }} \mathrm{s}$ generally were still closer to the true values than those obtained based on $n$ (eq. $13 \mathrm{a})$ or $n_{\text {deff }}$ (eq. 13b).

Results for $p_{\text {Low }}=0.20$ and $\theta=0.15$ were fairly typical and are discussed in most detail. ME based on use of equation 13a $(n)$ was 0.069; MAE was the same (to three decimal places) because all the errors were positive (Table 1), and $25 \%$ of the absolute errors were 0.083 or larger. With use of equation $13 \mathrm{~b}\left(n_{\text {deff }}\right), \mathrm{ME}=-0.063$, and the statistics for absolute errors were the same as for equation 13a (Table 1). Use of $v$ (eq. 13c) produced an ME of only -0.0027 and an MAE of 0.022 , considerably less than the corresponding values for equations $13 \mathrm{a}$ and $13 \mathrm{~b}$ (Table 1). Use of equations $13 \mathrm{~d}$ and $13 \mathrm{e}$ resulted in MAEs that were smaller than those statistics obtained from use of equations $13 \mathrm{a}$ or $13 \mathrm{~b}$, but larger than obtained from use of equation $13 \mathrm{c}$.

At $\theta=0.05$ (and $p_{\text {Low }}=0.20$ ), the errors arising from use of equations $13 \mathrm{a}$ and $13 \mathrm{~b}$ were smaller than at $\theta=0.15$ (Table 1 ), because at this low level of heterogeneity, the $p_{\text {High }}: p_{\text {Low }}$ relationship is only slightly different from the theoretical binomial relationship (Fig. 1A). All the prediction methods gave values of disease incidence similar to the true values. Nevertheless, use of equation $13 \mathrm{c}$, and even equations $13 \mathrm{~d}$ and $13 \mathrm{e}$, resulted in smaller errors than use of equation 13a or 13b. Larger errors were found for $\theta=$ 0.45 (and $p_{\text {Low }}=0.20$ ) when using equations $13 \mathrm{a}$ and $13 \mathrm{~b}$ (Table 1 ), because the $p_{\text {High }}: p_{\text {Low }}$ relationship is greatly affected by this level of heterogeneity (Fig. 1A). However, the predictions based on equation $13 \mathrm{c}$ were very close to the true values, with an ME of about -0.005 and an MAE of 0.022. MAEs based on $n$ and $n_{\text {deff }}$ were 0.114 and 0.087 , respectively. Predictions based on equations $13 \mathrm{~d}$ and $13 \mathrm{e}$ also were considerably closer to the true values than those based on equations $13 \mathrm{a}$ and $13 \mathrm{~b}$ (Table 1). ME, MAE, and $Q_{3}$ based on equation $13 \mathrm{c}$ were very similar in magnitude to the statistics for simulations at lower levels of heterogeneity (lower $\theta$ ).

At small $p_{\text {Low }}(=0.05)$, all the errors were small (Table 1$)$, because there is not much possible variation in predicted incidence at this $p_{\text {Low }}$. For instance, this simulation had the lowest $Q_{3}$ values of all the tested conditions. Errors based on equation $13 \mathrm{c}$ were still smaller than those based on equation $13 \mathrm{a}$ or $13 \mathrm{~b}$. The largest errors were for the simulations with $p_{\text {Low }}=0.40$. At this level of disease incidence, near the center of the 0 to 1 scale, there is a much wider range of possible predicted $p_{\text {Low }}$ values, which resulted in relatively large errors based on using equations $13 \mathrm{a}$ and $13 \mathrm{~b}$ (Table 1). Use of equation $13 \mathrm{c}$ at this large disease incidence still produced relatively small errors, and even over- or underestimation of $\theta$ (eqs. 13d and 13e) resulted in smaller errors than use of equation $13 \mathrm{a}$ or $13 \mathrm{~b}$.

\section{DISCUSSION}

The relationship between disease incidence at two levels in a spatial hierarchy is dependent on the degree of heterogeneity of disease at the lower level. Although the beta-binomial distribution can be directly used as a basis for relating probability of disease from one level to another $(21,33)$, use of this statistical distribution can be quite difficult, especially if one wants to predict the probability of disease at a lower level (e.g., leaf) based on probability of dis- 
ease at a higher level (e.g., plant). In the work reported here, an approximation method was developed for predicting probability of disease that uses an equation in the general form for the binomial distribution. In equation $1 \mathrm{c}$, number of individuals per sampling unit ( $n$ in eq. 1a) is replaced by a parameter $(v)$ that reflects the degree of disease heterogeneity at the lower level (as defined by the betabinomial aggregation parameter $[\theta]$ ). Equation $1 \mathrm{c}$ can be used to approximate the beta-binomial-based formula (eq. 2) under a wide range of realistic $n$ and $\theta$ values and requires no post-data-collection curve fitting. Selection of $v$ is done iteratively by matching various curves derived from the beta-binomial with the approximation-based curves (such as $\Delta p$ versus $p_{\text {Low }}$ [Figs. $2 \mathrm{~B}, 3 \mathrm{~B}$, and $6 \mathrm{~B}])$. Based on these results, an empirical equation was determined to predict $v$ based on $n$ and $\theta$ (eq. 10).

The use of $v$ in equation 1c has much in common with the use of Kish's (25) effective sample size $\left(n_{\text {deff }}\right)$. In the Kish approach (25), data are transformed so that the second moment (variance) of the discrete distribution of the data matches the theoretical second moment of a binomial distribution. The approach has been shown to recently be quite popular for many types of analyses based on overdispersed binary data $(6,38)$, since one can avoid use of some difficult statistical methods. With $v$ in this study, a parameter is selected so that the zero term of the discrete distribution of data (beta-binomial) matches the zero term of the theoretical binomial distribution. Therefore, $v$ can also be considered an effective sample size, because it allows one to use a binomial-based model to represent certain aspects of an overdispersed (clustered, aggregated) process.

There are some aspects of $v$ that should be considered for its proper application, especially in relationship to the use of $n_{\text {deff }}$. First, one should not substitute $v$ for $n_{\text {deff }}$ in the analyses discussed by Rao and Scott (36), because this would give wrong variances and, thus, lead to incorrect results. However, we have shown here that it is also incorrect to use $n_{\text {deff }}$ in the binomial-based formula for $p_{\text {High }}$. Specifically, use of equation $1 \mathrm{~b}$ with $\varepsilon=n_{\text {deff }}$ leads to underprediction of $p_{\text {High }}$ by about the same magnitude that use of $n$ in the standard binomial model (eq. 1a) leads to overprediction of $p_{\text {High }}$. Second, the zero term of the beta-binomial distribution (righthand side of eq. 2) cannot be exactly duplicated by $\left(1-p_{\text {Low }}\right)^{v}$. Exact duplication requires use of equation 1d, in which the exponent $(E)$ is a function of $p_{\text {Low }}$ and $\theta$ (eq. 11) (Fig. 5). Nevertheless, the approximation of using a single $v$ (eq. 1c) is very good under most realistic combinations of $n$ and $\theta$ (Fig. 3). The maximum difference between the beta-binomial $p_{H i g h}$ and the value predicted with equation 1c typically was less than 0.025. Evaluations of equation 1c using experimental data (Fig. 6) and simulations (Table 1) show that the approach is much more accurate and precise than use of equations based on the binomial distribution (eq. 1a) or on a binomial-type distribution with $n_{\text {deff }}$ replacing $n$ (eq. $1 \mathrm{~b}$, with $\varepsilon=n_{\text {deff }}$ ). Third, $\mathrm{v}$ is used in equation $1 \mathrm{c}$ to approximate only the zero term of the beta-binomial distribution. One could not approximate the full beta-binomial distribution (the probabilities of $X=1$ through $X=n$ diseased individuals per sampling unit) by using $v$ in the binomial formula, because no effort was made here to approximate the probabilities beyond the zero $(X=0)$ class. If one needed to represent the entire beta-binomial with a simpler expression, the approximation equations for generalized hypergeometric distributions shown on page 257 of Johnson et al. (24) may be of value. However, these full-distribution approaches do not necessarily predict the zero term of the beta-binomial distribution very well (L. V. Madden, unpublished data).

Comparison of equations 1c and 2 can help explain the impact of spatial pattern on disease incidence in a hierarchy. With a binomial distribution, individuals within sampling units are independent; that is, knowledge of the disease status of an individual provides no information on the disease status of other individuals within a sampling unit (32). For example, with $n=4$, the probability of no diseased individuals is calculated from equation $1 \mathrm{a}$ as the product of four terms

$$
\left(1-p_{\text {Low }}\right) \cdot\left(1-p_{\text {Low }}\right) \cdot\left(1-p_{\text {Low }}\right) \cdot\left(1-p_{\text {Low }}\right)
$$

On the other hand, as stated by Mak (32), spatial heterogeneity or overdispersion indicates that the individuals within a sampling unit are positively correlated (with an intracluster correlation of $\theta /[1+\theta]$ ). Viewed another way, this means that each sampling unit provides less than $n$ independent pieces of information about the disease status of the individuals within sampling units (5). With $n=4$, the probability of no diseased individuals can be calculated from equation 2 as the product of four terms

$$
\left(1-p_{\text {Low }}\right) \cdot\left(1-\frac{p_{\text {Low }}}{1+\theta}\right) \cdot\left(1-\frac{p_{\text {Low }}}{1+2 \theta}\right) \cdot\left(1-\frac{p_{\text {Low }}}{1+3 \theta}\right)
$$

The denominator of $p_{\text {Low }}$ gets larger with each multiplication term (e.g., $1,1+\theta, 1+2 \theta, \ldots$ ), which means that the terms in parentheses are successively increasing in value. This results in a larger value for the probability of no diseased individuals, or smaller $p_{\text {High }}$, compared with the binomial situation (Fig. 1A). Even though the product in the above calculation still comprises $n$ terms, the terms get larger to reflect the positive correlation in disease status of individuals within sampling units. That is, terms increase in value to account for the fact that knowledge of disease status of one individual tells us something about the disease status of subsequent individuals within a sampling unit. For instance, with $p_{\text {Low }}=$ 0.2 and $\theta=0.20$, the binomial result is $0.8 \cdot 0.8 \cdot 0.8 \cdot 0.8=0.41$ (or $p_{\text {High }}=0.59$ ) and the beta-binomial result is $0.800 \cdot 0.833$. $0.857 \cdot 0.875=0.50$ (or $\left.p_{\text {High }}=0.50\right)$.

The approximation of equation 1c operates to increase the probability of no diseased individuals in a sampling unit by keeping each term in the product the same $\left(1-p_{\text {Low }}\right)$ but reducing the number of terms in the product from $n$ down to $v$. In other words, because of the lack of independence of the individuals, the probability of no diseased individuals is determined by the product of fewer than $n$ terms. Thus, $n-v$ reflects, in some sense, how far the data are from random or how many pieces of independent data are available for calculation of $p_{\text {High }}$. This can most easily be visualized when $v$ is an integer (or approximately so). With the example of $n=4$ and $\theta=0.20, v=3.04$ (based on use of the Mathcad procedure), meaning that the probability of no disease individuals is given approximately by the product of three $(v \approx 3)$ identical terms, $0.8 \cdot 0.8 \cdot 0.8=0.51$ (or $p_{\text {High }}=0.49$ ). This is very close to the exact result of 0.50 shown above.

The emphasis in this article has been on the influence of heterogeneity on the $p_{\text {High }}: p_{\text {Low }}$ relationship (Figs. 1A and 6A). Given the quantified (or assumed) level of disease heterogeneity at the lower level, one can accurately predict the $p_{\text {High }}: p_{\text {Low }}$ relationship in order to estimate disease incidence at one spatial level from observed incidence data at the other spatial level. The method introduced in this article is based on equation 1c and the estimation of the parameter $v$, which is an effective sample size. Hughes and Gottwald (14) previously adopted the CLL transformation of equation 1a (eq. 5a) as a basis for this. One can consider equation 5a to be a special case of a more general model

$$
\ln \left[-\ln \left(1-p_{\text {High }}\right)\right]=\alpha+\beta \cdot \ln \left[-\ln \left(1-p_{\text {Low }}\right)\right]
$$

in which $\alpha$ and $\beta$ are parameters. For the binomial, $\alpha=\ln (n)$ and $\beta=1$ (eq. 5a). Hughes and Gottwald (14) fitted equation 5c to citrus virus data using a generalized linear model framework $(3,4)$ to determine empirically if equation $1 \mathrm{a}$, based on the binomial distribution, provided an appropriate description of the $p_{\text {High }}: p_{\text {Low }}$ relationship. Evidence of an inadequate description would be suggested by an estimated $\alpha$ not equal to $\ln (n)$ and an estimated $\beta$ not equal to 1 , either together or separately. Hughes and Gottwald (14) found for a data set with $n=4$ and $\hat{\theta}=0.116$ that estimates of $\alpha$ and $\beta$ were 1.2 and 1, respectively. Therefore, in this case, the back-transformation of equation $5 \mathrm{c}$ is not equation $1 \mathrm{a}$, but equation $1 \mathrm{c}$ with $\hat{v}=e^{\hat{\alpha}}(=3.3)$, since $\alpha$ represents $\ln (v)$ when $\beta=1$. 
Regarding equation 5c, several things can be noted. First, the approximation method discussed in the current article-which does not involve any curve fitting with observed data-provides a similar estimate of $v$. Equation 10 with $n=4$ and $\hat{\theta}=0.116$ gives $\hat{v}=3.26$. Second, our investigations of data sets for which the values of $n$ and $\hat{\theta}$ are much larger than those for the citrus virus data (14) indicate that it is rare to find $\beta=1$ (eq. 5c) (L. V. Madden and G. Hughes, unpublished data). When $\beta \neq 1$, the back-transformation of equation $5 \mathrm{c}$ is more complicated than the back-transformation of equation 5a. Third, equation 5c (with any value of $\beta$ ) is not expected to be satisfactory in the general sense if the beta-binomial is the appropriate distribution for data, because the $\operatorname{CLL}\left(p_{H i g h}\right)$ : $\operatorname{CLL}\left(p_{\text {Low }}\right)$ relation curves at large $p_{\text {Low }}$, especially for large $\theta$ (Fig. 1B), rather than being a straight line. Fourth, the flexibility of generalized linear modeling $(3,4)$ makes it is possible to modify equation $5 \mathrm{a}$ (or eq. $5 \mathrm{c}$ ) in other ways in order to deal with the overdispersion, such as substituting the function for $n_{\text {deff }}$ (eq. $9 \mathrm{~b}$ ) for $n$ in the CLL-based equation 5a. Although use of $n_{\text {deff }}$ directly does not predict $p_{H i g h}$ very well (Figs. 2A, 3A, and 6A), unless $n$ is small (Fig. 4C), one could fit this revised and expanded CLL model to observed data to obtain new estimates of $A$ and $b$ (and ignore the estimates of $A$ and $b$ obtained from the direct heterogeneity analysis of $\hat{p}_{\text {Low }}$ ). The equation can be written as

$$
\ln \left[-\ln \left(1-\hat{p}_{\text {High }}\right)\right]=\gamma_{1}+\gamma_{2}\left\{\ln \left[\hat{p}_{\text {Low }}\left(1-\hat{p}_{\text {Low }}\right)\right]\right\}+\ln \left[-\ln \left(1-\hat{p}_{\text {Low }}\right)\right]
$$

in which $\gamma_{1}=\ln \left(n^{b} / A\right)$ and $\gamma_{2}=1-b$. Estimates of $\gamma_{1}$ and $\gamma_{2}$ could be used to determine revised estimates of $A\left(A^{\prime}=\left(n^{1-\hat{\gamma}_{2}}\right) / e^{\hat{\gamma}_{1}}\right)$ and $b\left(b^{\prime}=1-\gamma_{2}\right)$ of the binary power law (15), which could then be used to obtain a revised $n_{\text {deff }}$ (eq. $9 \mathrm{~b}$ ) for predicting $p_{H i g h}$. However, when all that is required is an effective sample size for use in connection with a sampling program (14), the expedient solution is an analysis based on equation 1c as outlined in the current article, once one has obtained information, possibly from other studies, that the beta-binomial distribution describes the data at the lower level.

In conclusion, predicting disease incidence at one level from incidence at another level is possible in the presence of aggregation without using the complicated beta-binomial function. The derived approximation also leads to new understandings of the $p_{\text {Low }}: p_{\text {High }}$ relationship by showing how the lack of independence of diseased individuals approximately influences the probability of a sampling unit being diseased. Results will make it easier to use group sampling to estimate disease incidence at the lower level in a spatial hierarchy $(13,14,20)$.

\section{ACKNOWLEDGMENTS}

This research was conducted while L. V. Madden was on a research leave at the University of Edinburgh, Scotland, and supported by a Fellowship from the British Society of Plant Pathology (BSPP) and a grant from the Ohio State University Office of International Studies.

\section{LITERATURE CITED}

1. Ahn, H., and Chen, J. J. 1995. Generation of over-dispersed and underdispersed binomial variates. J. Comput. Graph. Stat. 4:55-64.

2. Campbell, C. L., and Madden, L. V. 1990. Introduction to Plant Disease Epidemiology. Wiley Interscience, New York.

3. Collett, D. 1991. Modelling Binary Data. Chapman \& Hall, London.

4. Crawley, M. J. 1993. GLIM for Ecologists. Blackwell Publishers, London.

5. Cussens, J. 1996. Effective sample-size in a dichotomous process with noise. Commun. Stat. Theory Methods 25:1233-1246.

6. Diabetes Control and Complications Trial Research Group. 1997. Clustering of long-term complications in families with diabetes in the Diabetes Control and Complications Trial. Diabetes 46:1829-1839.

7. Farrington, C. P. 1992. Estimating prevalence by group testing using generalized linear models. Stat. Med. 11:1591-1597.

8. Ferrandino, F. J. 1989. A distribution-free method for estimating the effect of aggregated plant damage on crop yield. Phytopathology 79:1229-1232.

9. Filipe, J. A. N., and Gibson, G. J. 1998. Studying and approximating spatio-temporal models for epidemic spread and control. Philos. Trans.
R. Soc. Lond. Ser. B Biol. Sci. 353:2153-2164.

10. Gubbins, S., and Gilligan, C. A. 1997. A test of heterogeneous mixing as a mechanism for ecological persistence in a disturbed environment. Proc. R. Soc. Lond. B Biol. Sci. 264:227-232.

11. Hepworth, G. 1996. Exact confidence intervals for proportions estimated by group testing. Biometrics 52:1134-1146.

12. Hughes, G. 1990. Characterizing crop responses to patchy pathogen attack. Plant Pathol. 39:2-4.

13. Hughes, G., and Gottwald, T. R. 1998. Survey methods for assessment of citrus tristeza virus incidence. Phytopathology 88:715-723.

14. Hughes, G., and Gottwald, T. R. 1999. Survey methods for assessment of citrus tristeza virus incidence when Toxoptera citricida is the predominant vector. Phytopathology 89:487-494.

15. Hughes, G., and Madden, L. V. 1992. Aggregation and incidence of disease. Plant Pathol. 41:657-660.

16. Hughes, G., and Madden, L. V. 1993. Using the beta-binomial distribution to describe aggregated patterns of disease incidence. Phytopathology 83:759-763.

17. Hughes, G., and Madden, L. V. 1995. Some methods allowing for aggregated patterns of disease incidence in the analysis of data from designed experiments. Plant Pathol. 44:927-943.

18. Hughes, G., and Madden, L. V. 1997. The determination of yield losses with aggregated patterns of diseased or missing plants. Pages 156-160 in: Exercises in Plant Disease Epidemiology. L. J. Francl and D. Neher, eds. The American Phytopathological Society, St. Paul, MN.

19. Hughes, G., and Madden, L. V. 1998. Comment: Using spatial and temporal patterns of Armillaria root disease to formulate management recommendations for Ontario's black spruce (Picea mariana) seed orchards. Can. J. For. Res. 28:154-158.

20. Hughes, G., Madden, L. V., and Munkvold, G. P. 1996. Cluster sampling for disease incidence data. Phytopathology 86:132-137.

21. Hughes, G., McRoberts, N., Madden, L. V., and Gottwald, T. R. 1997. Relationships between disease incidence at two levels in a spatial hierarchy. Phytopathology 87:542-550.

22. Hughes, G., McRoberts, N., Madden, L. V., and Nelson, S. C. 1997. Validating mathematical models of plant disease progress in space and time. IMA (Inst. Math. Appl.) J. Math. Appl. Med. Biol. 14:85-112.

23. Hughes, G., and Samita, S. 1998. Analysis of patterns of pineapple mealybug wilt disease in Sri Lanka. Plant Dis. 82:885-890.

24. Johnson, N. L., Kotz, S., and Kemp, A. W. 1992. Univariate Discrete Distributions, 2nd ed. Wiley Interscience, New York.

25. Kish, L. 1965. Survey Sampling. John Wiley \& Sons, New York

26. Madden, L. V., and Hughes, G. 1994. BBD-Computer software for fitting the beta-binomial distribution to disease incidence data. Plant Dis. 78:536-540.

27. Madden, L. V., and Hughes, G. 1995. Plant disease incidence: Distributions, heterogeneity, and temporal analysis. Annu. Rev. Phytopathol. 33: 529-564.

28. Madden, L. V., Hughes, G., and Ellis, M. A. 1995. Spatial heterogeneity of the incidence of grape downy mildew. Phytopathology 85:269-275.

29. Madden, L. V., Hughes, G., and Munkvold, G. P. 1996. Plant disease incidence: Inverse sampling, sequential sampling, and confidence intervals when observed mean incidence is zero. Crop Prot. 15:621-632.

30. Madden, L. V., Nault, L. R., Murral, D. J., and Apelt, M. R. 1995. Spatial pattern analysis of the incidence of aster yellows disease in lettuce. Res. Popul. Ecol. (Kyoto) 37:279-289.

31. Madden, L. V., and Nutter, F. W. 1995. Modeling crop losses at the field scale. Can. J. Plant Pathol. 17:124-137.

32. Mak, T. K. 1988. Analysing intraclass variation for dichotomous variables. Appl. Stat. 37:344-352.

33. McRoberts, N., Hughes, G., and Madden, L. V. 1996. Incorporating spatial variability into simple disease progress models for crop pathogens. Aspects Appl. Biol. 46:75-82.

34. Munkvold, G. P., Duthie, J. A., and Marois, J. J. 1993. Spatial patterns of grapevines with Eutypa dieback in vineyards with or without perithecia. Phytopathology 83:1440-1448.

35. Nyrop, J. P., and Binns, M. 1991. Quantitative methods for designing and analyzing sampling programs for use in pest management. Pages 67132 in: Handbook of Pest Management in Agriculture, Vol. 2. D. Pimentel and A. A. Hanson, eds. CRC Press, Boca Raton, FL.

36. Rao, J. N. K., and Scott, A. J. 1992. A simple method for the analysis of clustered binary data. Biometrics 48:577-585.

37. Shiyomi, M. 1981. Mathematical ecology of spatial pattern of biological populations. Bull. Natl. Inst. Agric. Sci. Ser. A Phys. Stat. 27:1-29.

38. Stewart, A. W., Asher, M. I., Clayton, T. O., Crane, J., Ellwood, P. E., Ford, R. P. K., Mitchell, E. A., Pattemore, P. K., and Pearce, N. 1997. The effect of season-of-response to ISAAC questions about asthma, rhinitis and eczema in children. Int. J. Epidemiol. 26:126-136.

39. Swallow, W. H. 1985. Group testing for estimating infection rates and 
probabilities of disease transmission. Phytopathology 75:882-889.

40. Tanne, E., Marcus, R., Dubitzky, E., and Raccah, B. 1996. Analysis of progress and spatial pattern of corky bark in grapes. Plant Dis. 80:34-38.

41. Turechek, W. W., and Madden, L. V. 1999. Spatial pattern analysis of strawberry leaf blight in perennial production systems. Phytopathology 89:421-433.

42. Upton, G., and Fingleton, B. 1985. Spatial Data Analysis, By Example. Vol. 1: Point Pattern and Quantitative Data. Wiley Interscience, Chichester, United Kingdom.
43. Waggoner, P. E., and Rich, S. 1981. Lesion distribution, multiple infection, and the logistic increase of plant disease. Proc. Natl. Acad. Sci. U.S.A. 78:3292-3295.

44. Xiao, C. L., Hao, J. J., and Subbarao, K. V. 1997. Spatial patterns of microsclerotia of Verticillium dahliae in soil and Verticillium wilt of cauliflower. Phytopathology 87:325-331.

45. Zarnoch, S. J., Anderson, R. L., and Sheffield, R. M. 1995. Using the $\beta$ binomial distribution to characterize forest health. Can. J. For. Res. 25: 462-469. 